\title{
Design and calibration of permanent magnet probes for the local measurement of velocity and temperature in a liquid metal backward facing step flow
}

\author{
Thomas Schaub $^{1}$ D $\cdot$ Sascha Wüstling ${ }^{2} \cdot$ Joachim Konrad ${ }^{3} \cdot$ Michael Tasler $^{4}$
}

Received: 30 June 2021 / Revised: 13 August 2021 / Accepted: 22 August 2021 / Published online: 29 September 2021

(C) The Author(s) 2021

\begin{abstract}
The simultaneous and local measurement of velocity and the temperature of a non-isothermal liquid metal flow has been an ongoing research topic over decades. The motivation is to obtain a detailed panorama of a liquid metal flow for the validation of turbulent heat flux models. So-called permanent magnet probes were used in the past for the local measurement of velocity and temperature profiles in liquid sodium in rather canonical flow configurations. The next step is to measure velocity and temperature profiles in a more complex flow geometry, namely a vertical confined backward facing step. For this, the permanent magnet probe must be adapted regarding its design, calibration procedure and temperature correction method. Particularly, considering that for this experiment the eutectic alloy of gallium, indium and tin was used as a working fluid, instead of liquid sodium, as in the mentioned past experiments. The main design aspects for a permanent magnet probe found in the literature are summarized and applied to the present probe. A calibration strategy for the probe was developed and implemented for the measurement of mean velocity profiles. A wetting procedure for the probe is proposed. The measured probe sensitivity for all six used probes agrees well with the theoretical estimations. The highest uncertainty contribution to measured sensitivity is related to the typical wetting issues of gallium-indium-tin. Future implementation of permanent magnet probes in general gallium-indium-tin experiments can make use of the developed know-how shared in this work.
\end{abstract}

\section{Graphic abstract}

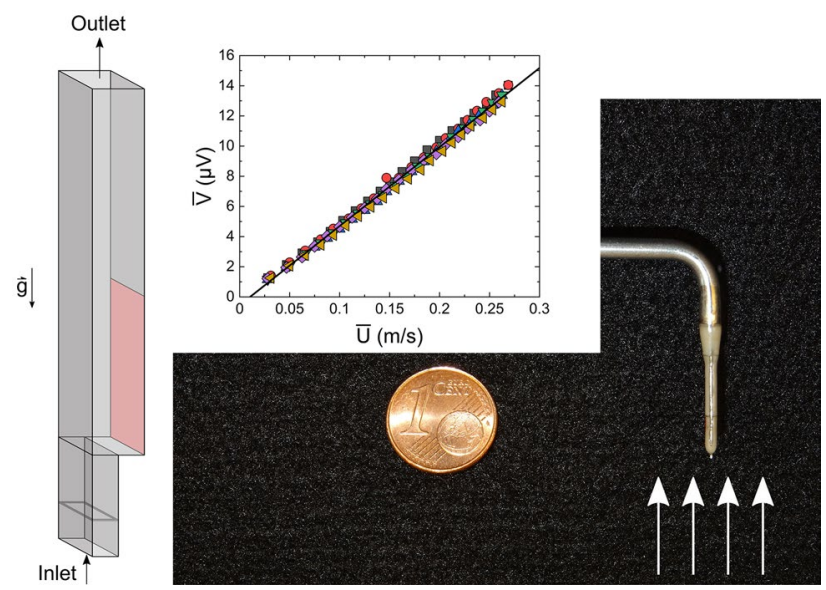

\section{Abbreviations}

BFS Backward Facing Step

CFD Computational Fluid Dynamics

Thomas Schaub

thomas.schaub@kit.edu

Extended author information available on the last page of the article
CFM Coriolis Flow Meter

DNS Direct Numerical Simulation

EMF Electro-Motive Force

GaInSn Gallium-Indium-Tin

GUM Guide to the Expression of Uncertainty in Measurement 


$\begin{array}{ll}\text { IFM } & \text { Inductive Flow Meter } \\ \text { JCGM } & \text { Joint Committee for Guides in Metrology } \\ \text { LIMCKA } & \text { Liquid Metal Competence Center Karlsruhe } \\ \text { PEEK } & \text { Polyether Ether Ketone } \\ \text { PMP } & \text { Permanent Magnet Probe } \\ \text { RANS } & \text { Reynolds-Averaged Navier-Stokes } \\ \text { RMS } & \text { Root Mean Square } \\ \text { SS } & \text { Stainless Steel } \\ \text { TC } & \text { Thermocouple } \\ \text { TFM } & \text { Turbine Flow Meter } \\ \text { UDV } & \text { Ultrasonic Doppler Velocimetry }\end{array}$

\section{Introduction}

There are several techniques for the measurement of local and global velocity-related quantities in a liquid metal flow (Eckert et al. 2007; Wetzel 2015). All have advantages and drawbacks that are to be assessed for each individual application. Ultrasonic Doppler velocimetry (UDV) is a wellestablished and probably the mostly used technique for the measurement of velocity profiles in liquid metals. Along its advantages one can mention its non-invasive character, the fact that with one single transducer one can measure a complete velocity profile with a very good spatial resolution, as well that they are commercially available. Besides, a lot of practical experience is available in the literature. When it comes to the measurement of turbulence related quantities, this measurement technique has the disadvantage of its relatively low its temporal resolution $f$. As can be inferred from the literature, $f$ is usually in the order of $f=O\left(10^{0}\right) H z$ (Zürner et al. 2018, 2020; Vogt et al. 2021), although higher temporal resolutions of a few $f=O\left(10^{1}\right) \mathrm{Hz}$ are possible — even for $2 D$ velocity-vector mapping (Franke et al. 2013). UDV can be complemented with thermocouples or thermocouple arrays to measure simultaneously velocity and temperature profiles. However, temperature sensors cannot be positioned at points along the sonic beam passing line. Furthermore, it is the local and simultaneous measurement at one point of mean and fluctuating quantities of both velocity and temperature fields that is of major interest for the validation of turbulent heat flux models for low Prandtl fluids.

As to the best knowledge of the authors, two measurement techniques fulfill the above-mentioned requirements: Pitot tubes with incorporated thermocouples and so-called permanent magnet probes (PMP). Historically, the Pitot tube alternative has been used in heavy-liquid metals while the permanent magnet probe in liquid sodium flows. The reasons are related to the different thermo-physical properties and the wetting capabilities of both liquid metal types.

Pitot tubes with incorporated thermocouples have the main advantage of using two well-known measurement techniques into one sensor. Due to the high density of heavy-liquid metals, the measurement of small pressure fluctuations with Pitot tubes is possible (contrary to the liquid sodium case). The use of these sensors for the measurement of turbulence related quantities is rather restricted by the limitations regarding their miniaturization: the smaller the sensor, the more complicated the task of guaranteeing a gasfree Pitot tube due to the high surface tension of heavy-liquid metals. Although there are technical solutions to this issue (Schulenberg and Stieglitz 2010), the state-of-art probes are still relatively large $(5 \mathrm{~mm})$.

Permanent magnet probes measure a velocity-proportional electric potential according to the known electromagnetic induction laws. If the voltage is not measured over regular wires (e.g., copper wires) but with thermocouples, the probe can simultaneously measure both the local velocity and the temperature of the flow. PMP have the main advantage that their miniaturization (i.e., its spatial resolution) is only limited by its components size and the used manufacturing techniques. Due to their working principle, the temporal resolution of the velocity signal transfer can be assumed as ideal. This, in the sense that electromagnetic time scales can be considered much faster than fluid mechanical time scales at the length scales that are resolved by the probe (Schulenberg and Stieglitz 2010). In other words, in the context of liquid metal flows, these probes are ideal for the study of turbulence related quantities. The main disadvantage of this measurement technique is that the induced velocity-proportional voltages are in the order of magnitude of only a few microvolts, i.e., two to three order of magnitude lower than a typical type $\mathrm{K}$ thermocouple temperature signal. Hence, the measurement task poses a real challenge regarding the suppression of electromagnetic interferences and signal conditioning. The reason why PMP have not been widely used in heavy-liquid metal applications is related to the poor wetting-capabilities of heavy-liquid metals. Major efforts were made in the past to counteract this problem with "sacrificial layers"- unfortunately, with no long-term success (Schulenberg and Stieglitz 2010). However, we believe to have found an alternative wetting technique that may be transferable to heavy-liquid metal flows (see Sect. 4.1). As to the best knowledge of these authors, Cramer et al (2013) is the only study that compared the performance of their permanent magnet probe design with UDV. They show that PMP are preferable over UDV to the study of turbulence related quantities. Although, to be fair, the UDV technique has improved considerably since the publication of Cramer et al. (2013), so a new comparison study would be a welcomed contribution to the literature.

PMP developed in the past were similar in design but varied in the used number of thermocouples and the thermocouple junction type. Depending on these differences, more or less complex calibration routines and velocity signal temperature 
correction methods need to be implemented. Also, the type of flow to be analyzed plays a role in the detail design of the probes, particularly, regarding its calibration and temperature correction and/or compensation methods. von Weissenfluh (1985) developed the first probes and used them in a sodium vertical pipe flow, von Weissenfluh and Sigg (1988) further developed their probe and applied it to a rod bundle flow, Knebel et al. (1994) used a different probe concept in a sodium vertical confined jet flow, while Kapulla et al. (2000) adapted his probe for a sodium horizontal mixing layer experiment. All these experiments were non-isothermal. Up to the best knowledge of the authors, there are no further PMP developments with thermocouples as electrodes available in the open literature.

In this work, a new PMP was designed and adapted to measure the local velocity and temperature of a vertical confined non-isothermal backward facing step (BFS) experiment with the eutectic alloy of gallium-indium-tin as the working fluid ( $G a I n S n)$. The results of the BFS experiment will be published in a separate paper. The probe design, the calibration and its temperature correction method had to be adapted to the present case. This work is a first step toward a similar experiment to be conducted in a large sodium facility (KASOLA facility), where the measurement of mean and fluctuating quantities of both velocity and temperature fields are the main objective.

This paper describes the measurement of local time-averaged velocities only. The measurement of the velocity and temperature variances plus the measurement of turbulent heat fluxes is technically possible with the developed probe. Due to time constraints and some minor technical optimizations to be made to the experimental facility, the treatment of fluctuating quantities could not be included at this stage of the project.

To put all probe design considerations into better context, the experimental facility will be presented first in Sect. 2. In Sect. 3, the developed permanent magnet probe is presented. Theoretical estimations for the probe sensitivity, the threshold for the appearance of nonlinear effects, the estimation of the influence of thermoelectric and thermomagnetic effects and the spatial and temporal resolution of the probe are discussed. Furthermore, the developed measurement chain is presented and the calibration procedure is introduced. Finally, the calibration results are shown together with the respective uncertainty analysis according to the Guide to the Expression of Uncertainty in Measurement (GUM) of the Joint Committee for Guides in Metrology (JCGM). The detailed uncertainty analysis is presented in the appendix.

\section{Experimental setup}

A sketch of the liquid metal experimental facility is shown in Fig. 1. The operational ranges expressed both in absolute and dimensionless terms are shown in the appendix in Table 2.
The working fluid is the eutectic alloy gallium-indium-tin $(G a I n S n)$. Its thermophysical properties for different temperature ranges are listed in the Appendix in Table 3. GaInSn has the advantage of being a non-toxic liquid metal at room temperature. It has an almost negligible vapor pressure, making its handling easy and safe, contrary to lead or lead-bismuth and particularly to liquid sodium. Along its disadvantages however one may mention its chemical incompatibility with aluminum, its price and its high surface tension. Wetting objects and surfaces with GaInSn is not an easy task. General handling guidelines with GaInSn can be found in Morley et al. (2008) and Cadwallader (2003). Long-term studies on its chemical compatibility with metals and plastics were recently published by Geddis et al. (2020).

The facility consists mainly four sections: the pumping-, the flow conditioning-, the test- and the return sections. All components but the pump and the flow meter were manufactured in-house. The facility has an height of approximately

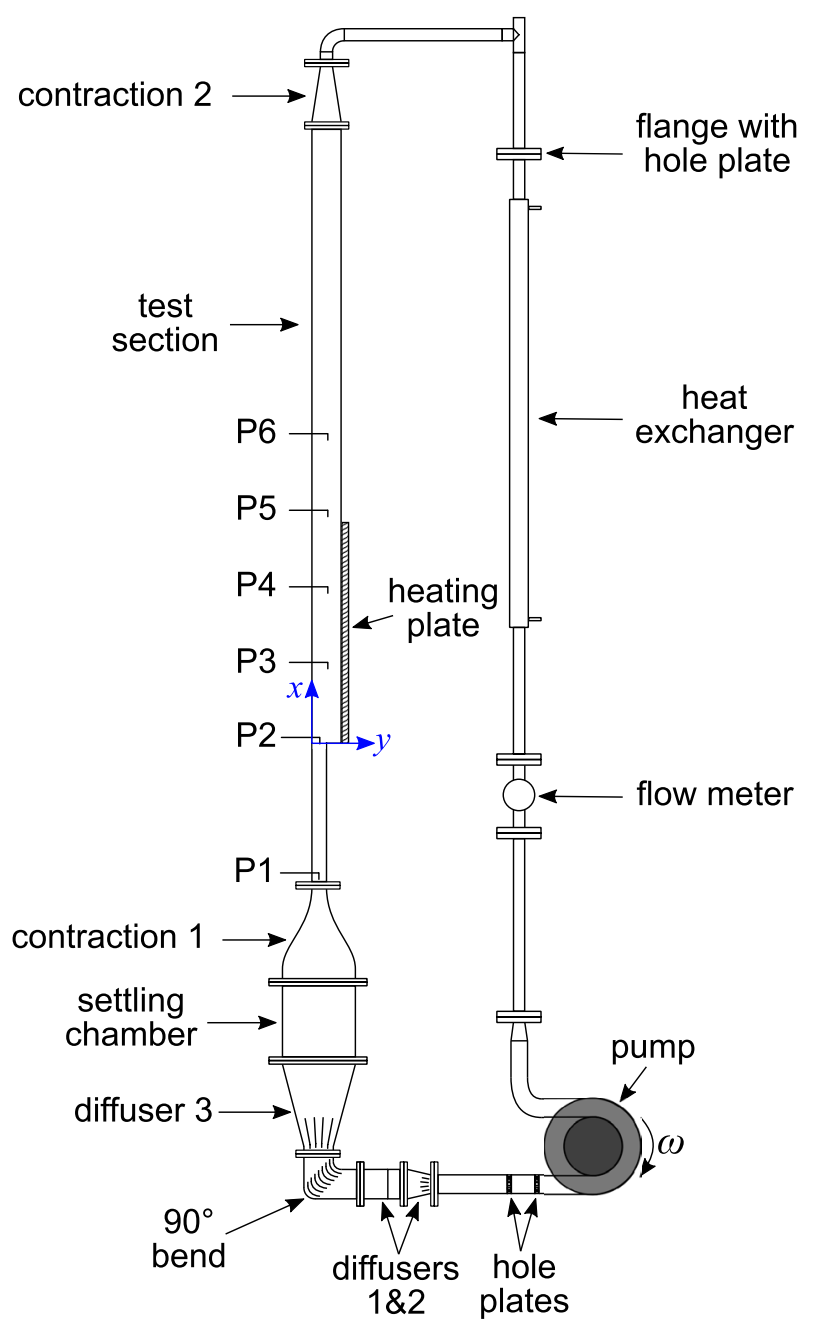

Fig. 1 Sketch of the experimental facility (to scale). Total height $3500 \mathrm{~mm}$ 
5 (m), including the storage tank (below the $90^{\circ}$ bend) and the expansion tank (following the vertical line of the heat exchanger, above the T-fitting).

The liquid metal is pumped with a SAAS GmbH disktype permanent magnet pump. The pump is driven by an electric motor which controlled by a Danfoss VLT digital variable frequency drive. Two type $T$ thermocouples are placed at the inlet and the outlet of the pump rectangular channel to measure the heat input of the pump to the liquid metal.

After the pump, the liquid metal flows through a flow conditioning section to correct for any upstream disturbances and to guarantee a flat constant inlet profile at the test section. The flow conditioning section consists of hole plates, wide angle diffusers with vanes and screens, a $90^{\circ}$ bend, a settling chamber with a honeycomb and three screen stages and a contraction. These components were designed using well-known wind- and water-tunnel design rules.

A confined backward facing step (BFS) test section with a heating plate right after the step is mounted into the facility. The test section was manufactured following a doublewall logic: an inner polyether ether ketone (PEEK) channel placed inside of and a stainless steel (SS) channel with the same shape. A trip-wire is placed a few step heights after the inlet to force the incoming boundary layer transition from laminar to turbulent at a single point. Otherwise, the laminar-to-turbulent transition point would be indefinite, generating undesired pressure fluctuations which would affect the flow downstream (Tihon et al. 2010). Along the test section, six in-house built permanent magnet probes are mounted which can be traversed along the $y$-axis with linear actuators. To measure the local wall temperature distribution on the contact surface between the heating plate and the GaInSn, 120 type $K$ thermocouples were inserted in a rectangular matrix into the heating plate.

The outlet of the test section is connected to the return piping with a contraction with a smooth transition from quadratic-to-round cross section. A cross-flow water/GaInSn heat exchanger removes the residual heat out of the liquid metal. The energy balance of the facility is monitored by measuring the liquid metal temperature before and after the heat exchanger. For the flow rate measurement, three flow meters with different operating principles were tested: an ABB Hygenic Master inductive flow meter (IFM), a NATEC NT turbine flow meter (TFM) and an EMERSON Micro Motion ELITE Coriolis flow meter (CFM). The IFM was mounted in series to the TFM and both were tested simultaneously. The CFM was tested separately due to space constraints. The comparison of the CFM and the IFM results remains a pending task.

The IFM was calibrated in an accredited laboratory in water. Due to the working principle of an IFM and considering that water and GaInSn have different electronic configurations (van der Waals forces versus electron cloud), the transferability of the IFM-calibration in water to $\mathrm{GaInSn}$ is not obvious. The idea behind installing the TFM in series to the IFM was to study the mentioned calibration-transferability. Unfortunately, the TFM did not work properly over longer periods of time. This, due to wetting issues of the rotor plain bearing with GaInSn. A comparison of the IFM with a flow meter with an alternative working principle was hence not possible. However, experience in partner research groups associated to the Liquid Metal Competence Center Karlsruhe (LIMCKA) gives confidence in the validity of the water-to-GaInSn calibration transferability. A comprehensive study is currently neither available here nor in the open literature.

\section{The permanent magnet probe and its measurement chain}

\subsection{Construction of the probe}

A picture of a PMP is shown in Fig. 2, whereas in Fig. 3 it is shown mounted into its housing as used in the experiment. A sketch of the probe is shown in Fig. 4 from different perspectives. The probe consists basically of five components: (i) a cylindrical shaped sheath made of PEEK which is connected to a stainless steel sheath. (ii) Inside the probe tip, an annular shaped permanent magnet with diametric magnetization direction is positioned so that the north pole of the magnet points in the $+y$ direction (Fig. 2). (iii) A thermocouple is passed through the permanent magnet and guided approximately $0.5 \mathrm{~mm}$ outside of the probe tip (TC3 in Fig. 2). (iv and v) Two further thermocouples are guided outside and along the probe tip from both sides ( $T C 1$ and $T C 2$ in Fig. 2). These thermocouples are electrically insulated to the liquid

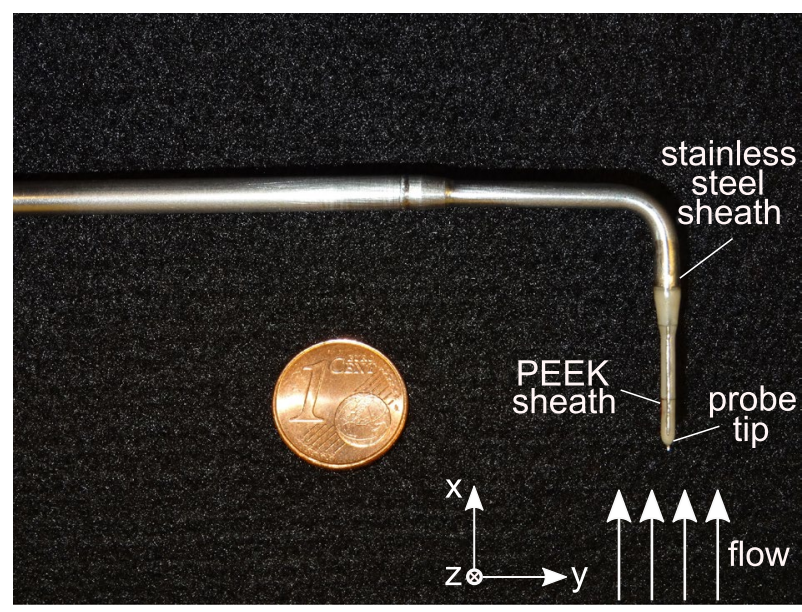

Fig. 2 Side-picture of a permanent magnet probe (PMP) without housing 

mounted in its housing
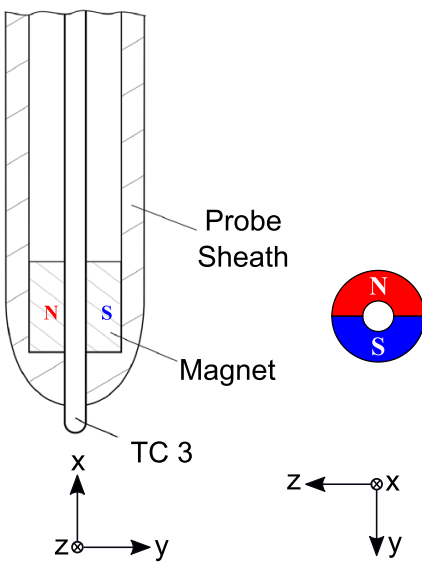

Fig. 3 Picture of the PMP
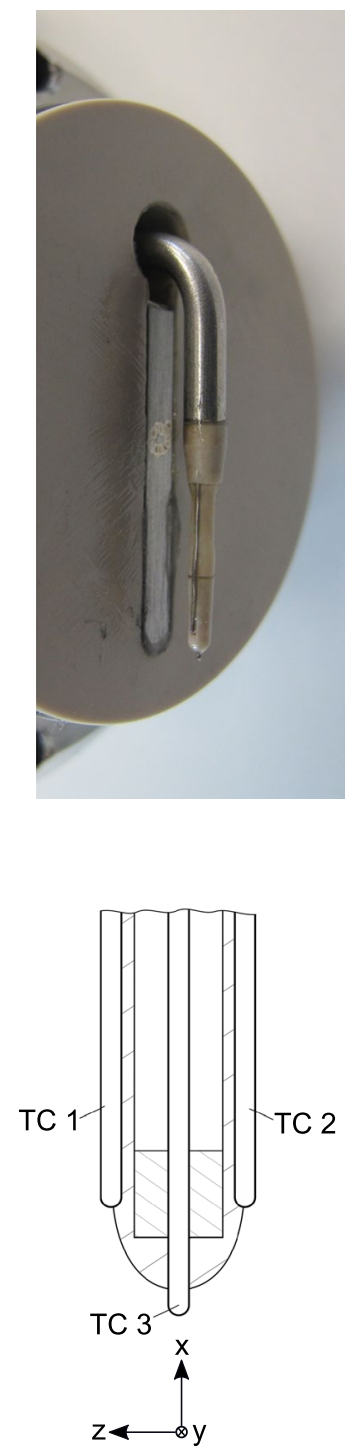

Fig. 4 Sketch of the PMP and its incorporated permanent magnet from different perspectives

metal with glue along their extension, except at their tips (Fig. 3). All three thermocouples are of type $K$, with an ungrounded measurement junction, an external diameter of $0.25 \mathrm{~mm}$ and a length of $500 \mathrm{~mm}$. All used thermocouples have a stainless-steel sheath (1.4401) and were manufactured from one batch by ThermoExpert GmbH. The thermocouples are guided through of the stainless steel shaft outside of the experiment. For the temperature measurement, not compensation but extension cables were used (all also from one batch). The magnet is a neodym $(\mathrm{NdFeB})$ permanent magnet with an external diameter of $d_{m}=1.0 \mathrm{~mm}$, a length equal to its diameter and a theoretical remanence of 1.4T. The probe tip external diameter is $d_{\text {tip }}=1.6 \mathrm{~mm}$. All components are glued together and electrically insulated from each other.
The reason for choosing type $K$ thermocouples for the probes was rather practical: at the time when designing the probes, ThermoExpert GmbH could only provide thermocouples with an outer diameter of $0.25 \mathrm{~mm}$ for type $\mathrm{K}$ thermocouples. Although thinner type $K$ thermocouples are commercially available, we preferred $0.25 \mathrm{~mm}$-thermocouples due to handling reasons since $0.10 \mathrm{~mm}$-thermocouples tend to break very easily.

\subsection{Principle of operation}

The probe works based magneto-hydrodynamics principles Davidson (2017). When an electrical conductive fluid particle flows with velocity $\vec{u}$ through a magnetic field $\vec{B}$, an electromotive force (EMF) is induced according to Faraday's law of induction. The EMF gives origin to a volumetric current density $\vec{J}$ according to Ohm's empirical law for conducting fluids,

$\vec{J}=\sigma(\vec{E}+\vec{u} \times \vec{B})$

,where $\sigma$ is the electrical conductivity of the liquid metal, $u \times B$ is the EMF induced due to the relative motion between the fluid particle and the magnetic field and $E$ may be an externally imposed electric field or-as in this case-is the resulting electric field due to the charge separation created by the EMF.

Applying Eq. (1) to the probe, one has the following situation: the liquid metal (an electric conductor) flows along the probe tip in the $+x$ direction (Fig. 2). The magnetization direction of the permanent magnet points in the $-y$-direction (Fig. 4). An EMF is induced according to the right hand rule in the $+z$-direction, although the voltage points in the $-z$ direction. The electromotive force pushes "positive" or "technical" particles" toward $T C 2$, making this the plus pole and $T C 1$ the minus pole. If two electric wires are placed within the induced electric field, the corresponding potential difference can be measured. In this case, since all thermocouples have an ungrounded measurement junction, the sheath of thermocouples TC1 and TC2 act as the two electrodes (Fig. 5). The sheath of TC3 is taken as the ground level for amplification purposes.

The thermocouples per se are used for local temperature measurement $T_{1}$ and $T_{2}$ (Fig. 5). They are also needed_for temperature-dependent corrections to be performed to $E$ in Eq. 1. In a flow with temperature gradients across the probe, thermoelectric and thermomagnetic electric fields are induced. The net electric field can be expressed as the superposition of the EMF proportional electric field $\vec{E}_{\text {ind }}$, an induced thermoelectric $\vec{E}_{\mathrm{th}, \text { el }}$ and thermomagnetic $\vec{E}_{\mathrm{th}}$, mag electric fields, i.e., $\vec{E}=\vec{E}_{\text {ind }}+\vec{E}_{\mathrm{th}, \mathrm{el}}+\vec{E}_{\mathrm{th} \text {, mag. }}$ Equation (1) is rewritten as 


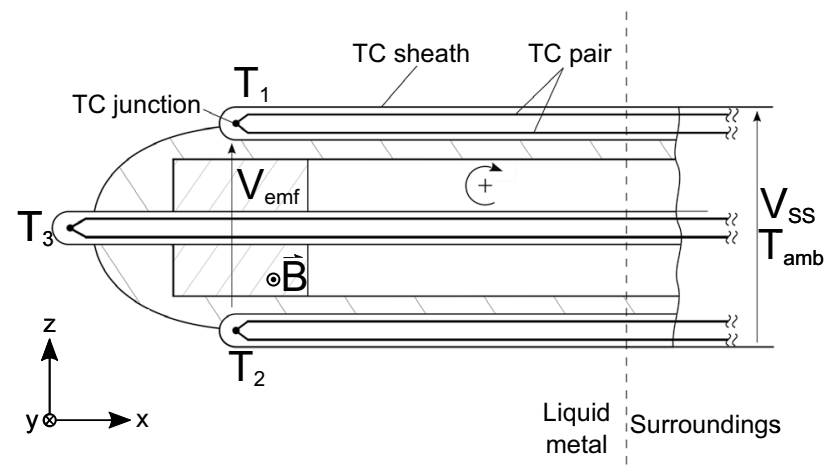

Fig. 5 Sketch of the PMP including relevant nomenclature from the electric diagram. Positive voltages are taken in clockwise direction

$\vec{J}=\sigma\left(\vec{E}_{\text {ind }}+\vec{E}_{\text {th, el }}+\vec{E}_{t h, \text { mag }}+\vec{u} \times \vec{B}\right)$

Alternatively, grounded thermocouples could have been also used as was the case in Knebel et al. (1994) and Kapulla et al. (2000). The velocity proportional induced voltage is measured in that case over the thermocouple-pair itself. On one hand, this has the advantage that the frequency response of the temperature signal is faster. On the other hand, it means that both temperature and velocity signals are overlapped and transferred in the same wires. Although those effects can be compensated by calibration (Knebel and Krebs 1994) or by correction (Kapulla et al. 2000), for this work it was decided to treat both velocity and temperature signals as individual signals at the expense of frequency response of the temperature signal. This is one of the key difference between this probe and probes developed in the past.

\subsection{Measurement equation}

The first step to obtain the measurement equation is to take the divergence of Eq. (2):

$\nabla \cdot \vec{J}=\nabla \cdot(\sigma(\nabla \phi+\nabla \psi+\vec{u} \times \vec{B}))=0$

where the fluid velocity proportional electric field and the induced thermoelectric field are expressed in terms of gradients of respective potential fields, i.e., $\nabla \phi=E_{\text {ind }}$ and $\nabla \psi=E_{\text {th }}$, el. The equation is set to zero because of the conservation of charge for conductors moving at speeds way below the speed of light (Davidson 2017).

In a second step, the variables expressed in Eq. (3) must be related to quantities that can be measured in practice. This is done by means of Green's functions (Kapulla 2000). As a result, a general measurement equation for a probe can be obtained. For this particular probe, important simplifications can be made, since all probe components are electrically insulated each other. Taking those simplifications into consideration, using the nomenclature of Fig. 5 and using Kirchhoff's circuitry laws, the measurement equation for this probe reads

$$
\begin{aligned}
& \mathrm{Ku}_{\mathrm{vol}}+S_{\mathrm{SS}}\left(T_{\mathrm{amb}}-T_{1}\right)-V_{\mathrm{SS}}+S_{\mathrm{SS}}\left(T_{2}-T_{\mathrm{amb}}\right) \\
& \quad+S_{e, \mathrm{tip}}\left(T_{1}-T_{2}\right)=0
\end{aligned}
$$

where $K$ is the probe sensitivity, $\mathrm{u}_{\mathrm{vol}}$ is the fluid volumeaveraged velocity in the volume of influence of the permanent magnet (to be discussed more in detail in Sect. 3.4.7), $\mathrm{K} \mathrm{u}_{\mathrm{vol}}$ is the velocity-proportional induced component of the signal, $S_{\mathrm{SS}}$ the Seebeck-coefficient of stainless steel (SS), $T_{1}$ and $T_{2}$ the temperature of the liquid metal at points 1 and $2, T_{\mathrm{amb}}$ the ambient temperature of the thermocouple connectors outside of the test section where the velocityproportional induced voltage $V_{\mathrm{SS}}$ is measured and $S_{e, \text { tip }}$ is the effective Seebeck-coefficient of the probe tip. Depending on the probe design, $S_{e \text {,tip }}$ must be determined either by calibration or, if the probe tip components are electrically insulated from each other, $S_{e, \text { tip }}$ can be set equal in value as the Seebeck-coefficient of the liquid metal equal. $T_{a m b}$ is assumed to be equal for both thermocouple connectors (see Sect. 3.4.3).

Rearranging Eq. (4) one finally gets

$V_{\mathrm{SS}}=\mathrm{Ku} \mathrm{vol}_{\mathrm{vol}}+S_{e}\left(T_{2}-T_{1}\right)$,

where $S_{e}=\left(S_{\mathrm{SS}}-S_{e \text {,tip }}\right)$ can be interpreted as an effective Seebeck-coefficient for the probe and the thermocouple sheaths.

In conclusion, the measurement task for the determination of the local volume-averaged velocity of the fluid $u_{v o l}$ consists of measuring the actual induced voltage $V_{\mathrm{SS}}$, obtaining the probe sensitivity $K$ by calibration and measuring the temperatures $T_{1}$ and $T_{2}$ across the probe for correcting for any imposed thermoelectric effects.

\subsection{A priori considerations when designing the probe}

The following design aspects are valid for any kind of permanent magnet probe-independent of its application. It is the calibration of the probe and the needed temperature corrections to be made that may vary from case to case.

\subsubsection{Theoretical estimation of the probe sensitivity}

The key parameter in Eq. (5) for calculating the local flow velocity from the measurement of $V_{\mathrm{SS}}$ is the probe sensitivity $K$. Assuming for the sake of simplicity that the permanent magnet is a sphere and further assuming that the probe components and flow alignment is perfect, a theoretical estimate 
for the probe sensitivity $K_{\text {th }}$ can be calculated. Kapulla (2000) shows that this expression reads,

$K_{\mathrm{th}}=\frac{1}{2} B_{0} \frac{R^{3}}{r^{2}}\left(1+\frac{\sigma_{f}-\sigma_{s}}{2 \sigma_{f}+\sigma_{s}}\right)$

, where $B_{0}$ is the magnetic flux density of the permanent magnet on the magnet surface, $R$ is the permanent magnet radius, $r$ the distance between the sphere center and one of the electrodes, $\sigma_{f}$ the electric conductivity of the fluid and $\sigma_{s}$ the net electric conductivity of the probe tip (probe tip sheath plus permanent magnet).

As mentioned before, in this case the permanent magnet is electrically insulated from the liquid metal and electrodes. Furthermore, the probe tip is made of PEEK, i.e., $\sigma_{s}=0$. Before mounting the permanent magnets into the probe tip, $B_{0}$ was measured for every used permanent magnet with a small Hall sensor placed on the north pole of the magnet. A rough average value for all used permanent magnets is $B_{0} \approx 0.35 T$. Considering that the thermocouples at the probe tip have a separation $d_{T C}$ of $d_{T C} \approx d_{t i p}=1.6 \mathrm{~mm}$, i.e., $r=0.8 \mathrm{~mm}$, that the external diameter of the permanent magnet is $d_{m}=1.0 \mathrm{~mm}$, i.e., $R=0.5 \mathrm{~mm}$ and taking the electric conductivity of $\operatorname{GaInSn}$ at $25^{\circ} \mathrm{C}$ as $\sigma_{f}=3.25 \times 10^{6} 1 /(\Omega \times m)$ (see Table 3), one obtains $K_{\text {th }}=51 \frac{\mu V}{\mathrm{~m} / \mathrm{s}}$. As will be seen in Sect. 4.4, this estimated value for $K_{t h}$ agrees well with the measured sensitivities. Taking $K_{\text {th }}$ together with the estimated velocities in the experiment, one may calculate the effective induced voltages.

\subsubsection{Deviation of the probe sensitivity from linearity}

The next step is to assess up to which extent it is possible to assume a linear response of the probe signal. For this, one has to take a closer look to the underlying physics.

As was discussed in Sect. 3.2, the relative motion of a fluid particle in a magnetic field $\underline{B}$ induces a EMF, which in turn induces a current density $J$. The charged particles of $J$ induce a further induced magnetic field $B_{\text {ind }}$ according to Ampere's law. $B$ ind interacts with the magnetic field $B$. However, if $J \approx 0, B$ ind can be neglected. Whether this is the case or not is given by the magnetic Reynolds number

$\operatorname{Re}_{m}=\mu_{s} \sigma U_{c} l_{c}$,

where $\mu_{s}$ is the permeability of free space, $\sigma$ the fluid electrical conductivity $U_{c}$ the characteristic velocity of the liquid metal and $l_{c}$ the characteristic length of interest of the liquid metal (in this case the spacing between both wires). For flows where $\operatorname{Re}_{m} \ll 1$, one may assume that $B$ ind is negligible. For this case, $l_{c}=1.6 \mathrm{~mm}$. The maximum possible bulk velocity $U_{b}$ for this experiment is $U_{b}=U_{c}=0.247 \mathrm{~m} / \mathrm{s}$. Substituting these values into Eq. (7) one obtains $\mathrm{R} e_{m} \sim 0.006$.
This means that an eventual nonlinear behavior of the probe sensitivity must be explained by other means.

A second phenomenon that plays an important role is that of the interaction between the induced Lorentz force and the inertial forces of the fluid particles passing by the probe tip. As it is known, Lorentz forces oppose to the relative motion of the fluid particles. That is, if the fluid inertia is below a certain threshold, it may be expected that the induced Lorentz forces distort the flow structure in the vicinity of the probe tip. The dimensionless parameter that defines this threshold is the so-called interaction parameter $N$ (Davidson 2017) which reads

$N=\frac{\sigma \bar{B}_{0}^{2} l_{c}}{\rho U_{c}}$

, where $\rho$ is the fluid density and $\bar{B}_{0}$ is the volumetric average of the magnetic field density around the probe tip.

One has to consider that for the calculation of the interaction parameter $N$ one should not use the measured value of $\vec{B}$ at the permanent magnet surface $B_{0}$ but some sort of volume-average for $B$ around the probe tip $\bar{B}_{0}$ (as indicated in Eq. (8)). One alternative for calculating $\bar{B}_{0}$ is by taking Eq. (1) and assuming $J$. One obtains,

$B_{0} \approx \frac{\Delta \phi}{\Delta l} \frac{1}{U_{b}}=\frac{\Delta \phi}{U_{b}} \frac{1}{\Delta l}=K \frac{1}{\Delta l}$

where $\Delta \phi$ is the potential difference between the two electrodes separated by $\Delta l$ and $\Delta \phi / U_{c}$ can be understood as the sensitivity of the probe. For $K$ either a measured or the theoretical sensitivity can be used. The assumption $J \approx 0$ for permanent magnet probes was empirically validated by Ricou and Vives (1982).

Although there is no defined hard threshold for $N$, let's say that the Lorentz force should play a role when $N \sim 1$. The lowest bulk velocity that can be generated in this experiment is $U_{b}=0.016(\mathrm{~m} / \mathrm{s}$ ) (Table 2, Appendix $)$. Taking $U_{b}, \quad \sigma=3.25 \times 10^{6} 1 /(\Omega \times m)$, $B_{0} \approx K_{t h} / \Delta l=51 \times 10^{-6} \frac{\mu V}{\mathrm{~m} / \mathrm{S}} / 0.0016 \mathrm{~m}=0.032 T \quad$ an $l_{c}=1.6 \mathrm{~mm}$, one obtains $N=0.051$. Now, if one sets $N=1$ in Eq. (8), one obtains a threshold for the bulk velocity $U_{b}=U_{c}<0.83 \mathrm{~mm} / \mathrm{s}$. Due to limitations regarding a stable operation of the experimental facility pump at very low flow rates, this threshold could not be experimentally validated. However, Cramer et al. (2006) report nonlinear behavior for a similar probe in a GaInSn experiment for velocities $U_{c}<1 \mathrm{~mm} / \mathrm{s}$. Taking the previously estimated sensitivity for the present probes of $K_{\mathrm{th}}=51 \frac{\mu \mathrm{V}}{\mathrm{m} / \mathrm{s}}$ this would imply an induced voltage in the order of $V_{\mathrm{SS}} \sim 0.042 \mu \mathrm{V}=42 \mathrm{nV}$.

A further aspect to take into consideration is the volumeaveraged velocity profile around the probe tip. Depending on the thickness of the boundary layer around the probe tip, 
nonlinear effects can also be expected. There is no reason to expect a perfectly linear response of the probe along all velocities. However, as will be shown later, for the covered range in this experiment, a linear sensitivity curve is a good approximation.

\subsubsection{Thermoelectric effects}

When using permanent magnet probes in non-isothermal experiments, one needs to consider thermoelectric effects that may appear during the experiment.

Assume that a thermal gradient exists over the probe tip and that the wires are not only electrically connected over the liquid metal, but also over an electrically conducting probe sheath and the permanent magnet (as for past probe designs). Thermoelectric voltages are hence induced along the probe tip. These induced thermoelectric Seebeck-voltages can be treated as an effective Seebeck-coefficient $S_{e, t i p}$ for the probe tip. Kapulla (2000) derived an expression to estimate $S_{e, t i p}$ which reads,

$S_{e, \mathrm{tip}}=S_{f}\left(\frac{\sigma_{s} S_{s} / \sigma_{f} S_{f}+1}{\sigma_{s} / \sigma_{f}+1}\right)$

, where $S_{f}=S_{\text {GaInSn }}$ is the Seebeck coefficient of the fluid (in this case the liquid metal) and $S_{s}$ is the Seebeck coefficient of the solid (in this case the probe tip).

When $\sigma_{s}=0$, i.e., in the case of an electrically insulating probe tip (as in this case), one has

$S_{e, \mathrm{tip}}=S_{f}=S_{\text {GaInSn }}$

, where $S_{\text {GaInSn }}$ is the Seebeck-coefficient of GaInSn.

In the case that $\sigma_{s} \neq 0, S_{e}$ must be determined by calibration (Knebel and Krebs 1994; Weissenfluh 1985) or by complex temperature compensation methods Kapulla et al. (2000). This is a further key advantage of the present probe design compared to the ones used in the past.

The influence of the thermoelectric effects depends on the local temperature gradients along the probe tip, as well as on the respective Seebeck-coefficients (see Eq. (5)). For this experiment, the maximum measured relative thermoelectric contribution to $V_{S S}$ was $\approx 2.5 \%$. For past probe developments in liquid sodium experiments, the influence of this correction term was-depending on the measurement position and parameter set—of the same order of magnitude as the measured signal.

Further, non negligible thermoelectric effects may also be induced in the liquid metal by macro thermal gradients along the test section. For this experiment, these may appear along the $y$-axis, i.e., the temperature difference between the inlet bulk flow and the fluid temperature at the heating plate. It is then fair to assume that any macro thermoelectric effects can be neglected by placing the probe wires or thermocouples perpendicularly to the thermal gradients and by considering the small thermal gradients in this experiment.

On top of any thermoelectric effect that may appear within the experimental facility, one needs to take into consideration all external thermo-electric effects that may influence the signal outside of the test section. The experimental facility is located in a wide and large experimental hall. Of course, thermal stratification of the ambient air is present, as well as air currents when gates and/or doors are opened and closed. Experience showed that not negligible thermoelectric effects were induced by these effects at the thermocouple connectors of the probe (as well as for all other thermocouples). This was solved by housing the thermocouple connectors of each probe within an aluminum box and by insulating it with $\sim 20(\mathrm{~mm})$ thick insulating material (Armaflex ${ }^{\circledR}$ ). Furthermore, all thermal gradients along the thermocouples had to be minimized as well, particularly at those places were plastic deformation of the thermocouple could be observed (Fenton (1980)). The thermocouple cold junction (ISOTECH TRU937) was placed into an enclosure inside the control room to reduce down to a minimum any influence of external temperature fluctuations.

\subsubsection{Thermomagnetic effects}

Along all possible thermomagnetic effects, only the Nernst-Ettingshausen plays a role for this type of probe (Kapulla, 2000). The relation of the Nernst-Ettingshausen thermomagnetic potential has the form $V_{\text {th,magn }} \propto \nabla T \times B$. That is, by simply placing $B$ parallel to the possible larger thermal gradients, this effect may be neglected, as it is in this case.

Regarding the temperature gradients in stream-wise direction, Kapulla (2000) shows that even for a sodium experiment with much larger thermal gradients than the present one, this contribution is no larger than $\approx 2(n V)$. In other words, thermomagnetic effects are even lower than the thermal noise of the used electronics. This effect may only play an important role in MHD applications with very large magnetic fields.

The use of type $K$ thermocouples should be avoided in MHD applications under large magnetic fields and large temperature gradients. Barleon et al. (1996) show that the induced errors under the mentioned circumstances are not negligible, hence type $T$ thermocouples should be used instead. Knebel and Krebs (1993) showed empirically that for type $K$ thermocouples the influence of the permanent magnet on the probe sensitivity is indeed negligible. 


\subsubsection{Probe orientation and its influence on the flow}

From $\vec{J}=\sigma(\vec{E}+\vec{u} \times \vec{B})$ it becomes evident that there is directional dependency between the induced signal, the magnet orientation and the flow direction. Ricou and Vives (1982) validated empirically the theoretical directional dependency for a PMP. For this experiment, design measures were taken to avoid any larger deviations from the ideal conditions (perfect perpendicular alignment between the three vectors $E, \vec{u}$ and $B$ ). All probe tip components were manufactured with tolerances of $0.2(\mathrm{~mm})$, while for its mounting under the microscope, the alignment of the parts was kept below $1^{\circ}$. Considering that the probe sensitivity must be determined experimentally, any directional deviation from ideal conditions for the streamwise velocity component will be contained in the probe sensitivity. This, of course, only under the flow conditions where the calibration was made. However, in a highly three-dimensional flow as it is in a confined BFS, all three velocity components may induce a signal component, if the alignment of the parts deviates too much from their ideal alignment. Due to the nature of $\vec{J}=\sigma(\vec{E}+\vec{u} \times \vec{B})$, the error associated with signal components induced by the velocity components perpendicular to the streamwise component can be considered $<1 \%$, as long as the alignment of the probe tip components is kept $<1^{\circ}$ (von Weissenfluh 1985). To check the directional dependency of each manufactured probe is not a trivial task. For this, a separate experiment would be necessary, which was out of the scope of this work, but is definitely a pending task. Particularly-as already mentioned-considering the complex threedimensional nature of a confined BFS.

The used probes are invasive, although the probe itself has a relatively small diameter. The probe shaft was manufactured thicker, on one hand, to be able to hold against any deflection due to the flow, and on the other, due to a purely practical reason. The stainless steel probe shaft diameter had to be chosen in such a way for the leak prevention system of the probe housing to work properly. The design of a liquid metal leak-free system with moving parts (in this case an axial displacement) is not a straightforward task. Particularly, considering that for this facility no bellow-system could be used due to the limited amount liquid metal in the lab.

The ratio between the displacement area of the probe tip and the inlet cross section area is $0.063 \%$. For the outlet cross section area it is $0.031 \%$. The distance between the probe tip and the stainless steel shaft is $30 \mathrm{~mm}=18.75 d_{\text {tip }}$. The influence of the probe tip on the flow is then assumed to be negligible.

\subsubsection{Time lag for velocity fluctuations}

Equation (5) is the general measurement equation for the instant velocity of the flow. Since it contains a correction term for thermoelectric effects, one has to consider the dynamic response of the thermocouples, if velocity and temperature fluctuations are of interest. In the present case, due to time constraints of the project, the study of velocity fluctuations was not done. Kapulla et al. (2000) and von Weissenfluh (1985) present strategies on how to account for these issues.

\subsubsection{Spatial and temporal resolution of the probe}

Finally, the question about the definition of the local and instantaneous character of the measurements, i.e., the spatial and temporal resolution of the probe must be addressed.

The probe velocity-proportional signal $V_{S S}$ is a consequence of the induced $E M F$ according to $\vec{J}=\sigma(\vec{E}+\vec{u} \times \vec{B})$. The induced $E M F$ - and hence the measured velocity $\vec{u}$ - has a volumetric character, in the sense, that the EMF can be measured along two points within the volume enclosed by the volume of influence of the magnetic field $B$. Depending on how close the electrodes are positioned to the permanent magnet surface, the $E M F$ will be stronger or weaker. This only has an influence on the probe sensitivity $K . V_{S S}$ is related to the velocity $\vec{u}$ by calibration. The measured velocity $\vec{u}$ correspondsconceptually - to the average velocity of a fluid bulb (or eddy) of the size of the volume of influence of $\vec{B}$, as if the probe wasn't there. This, since the probe influence on the flow itself and/or any permanent magnet positioning deviation are contained within $K$. The probe resolution is then assumed to be the distance between both electrodes. Whether this assumption is justified or not, must be experimentally determined. However, this assumption seems justified considering that the magnetic field density decreases from the permanent magnet surface with distance as $1 / r^{2}$. The temporal resolution of the probe can then be estimated assuming Taylor's hypothesis of frozen turbulence taking the probe diameter as the spatial resolution and the local fluid velocity, i.e., for $d_{t i p}=1.6 \mathrm{~mm}$ and the maximum inlet bulk velocity for the calibration campaign $U_{b}=0.247 \mathrm{~m} / \mathrm{s}$, the temporal resolution can be estimated as $154 \mathrm{~Hz}$.

The validation of the estimated temporal resolution of the probe and/or the measurement chain has not been measured so far and remains an important pending task. However, one has to distinguish between the frequency response of the measurement chain per se and the intrinsic spatial low-pass filtering that the probe has due to its finite dimension. As mentioned before, it is actually not the probe diameter that low-passes the signal, but the volume of influence of the permanent magnet. Kapulla et al. (2000) determined the temporal resolution of their probe by comparing the measured 
probe signal FFT with the FFT of a LDA-signal measured in a hydraulically similar water experiment.

The question about the relationship between the frequency spectra and the wavenumber spectra of a turbulent flow, under which conditions it may make sense to relate both and how meaningful is the study of the frequency spectra at all, can be found in George (2013). For the present geometry, we think that a wavelet analysis may be the right approach to follow to study any frequency related phenomena (Farge (1992), Farge et al. (1996)).

\subsection{Data acquisition system and suppression of electromagnetic interference}

The actual experiments were made at Reynolds numbers based on the step height and the inlet bulk velocity at $\operatorname{Re}_{h} \sim 10000, \sim 20000$ and $\sim 30000$. The estimated probe sensitivity is $K_{\text {theory }}=51 \frac{\mu \mathrm{V}}{\mathrm{m} / \mathrm{s}}$ while the bulk velocity range for the present experiment of $0.042<U_{\text {bulk }}<0.247 \mathrm{~m} / \mathrm{s}$. The velocity range corresponds to the minimum outlet bulk velocity for $R e_{h} \sim 10000$ and the maximum to the inlet bulk velocity for $\mathrm{Re}_{h} \sim 30000$. The theoretical signal range can then be estimated. This means that the mean probe signals for the bulk velocities were expected to be within the range of $2.14<\bar{V}_{\mathrm{SS}}<12.60 \mu \mathrm{V}$. Of course, turbulent fluctuations two orders of magnitude lower should also be captured by the measurement system. To put these values into perspective, a typical DC voltage generated by a thermocouple is in the order of a few $1000 \mu V=1 \mathrm{mV}$.

In other words, there were two factors determining the success of this measurement task. First, the design of a very low noise measurement chain and, second, being able to suppress external electromagnetic interferences source down to a minimum.

\subsubsection{Measurement system}

The main challenge was to find a commercially available measurement system capable of reaching the limit of what is technically possible. The chosen measurement system was an LTT24 from Labortechnik Tasler GmbH with an included Linear Technology LT6018 operational amplifier. The probe signal $V_{S S}$ enters the differential ended mode channel with a range of $\pm 5 \mathrm{mV}$. For this particular task, the standard LTT24 differential amplification stage $(20: 1)$ was preceded by a further amplification stage $(50: 1)$ with an LT6018 operational amplifier (soldered into the LTT24 system). The amplified signal is digitized immediately with a $24 b i t$ A/D converter which oversamples the signal with $32 \mathrm{MHz}$. The digital signal goes into a noise-shaping routine and is finally transferred at a decimation at rate of $5 \mathrm{kHz}$ to the PC. Finally, the signal is digitally low-pass filtered with an 8-pole
Butterworth filter at $200 \mathrm{~Hz}$ for mean velocity measurements and at $500 \mathrm{~Hz}$ for time-resolved signal measurements.

\subsubsection{Suppression of electromagnetic interference}

A very low noise measurement is not only a challenge regarding the measurement chain equipment, but also regarding the strategy for the suppression of electromagnetic interference. Since the pump was driven by a variable frequency drive, the external electromagnetic interference influence on the probe signal was massive. Initially, the useful signal completely disappeared within the picked-up electromagnetic interference from the pump variable frequency drive.

The problem was solved, first, by selecting short and premium quality shielded and twisted cables for the probe. Second, by closing existing interruptions in the cable shielding network. This may seem to create ground loops at first, but this is not the case. Instead, it completes a closed Faraday cage along the signal path (Fig. 6). Third, by grounding all cables to and from the variable frequency drive on both sides and by lowering the variable frequency drive switching frequency to its minimum. And fourth, by choking common-mode interference currents by means of Würth Elektronik clamp-on ferrites for low frequencies. The probe cables were coiled around and then passed through ferrites. All cables connected to the variable frequency drive were passed through ferrites. Further noise rejection strategies for a similar probe can be found in Cramer et al. (2006).

A rough estimation of the noise level due to intrinsic physical phenomena of any measurement system can be found in the Appendix 9.2. The calculations for the measurement system alone (i.e., without plugging the probe to the LTT24) indicate that achieved noise level was below the mentioned theoretical worst-case threshold for thermaland flicker noise contributions. When plugging the probe to the LTT24, the external electromagnetic interferences were suppressed down to $\sim 80 \mathrm{nV}$ (representative estimate for all channels and probes).

\subsection{Comparison of the present probe design compared to probes used in the past}

Now that all design considerations were mentioned, the advantages and disadvantages of the present probe design compared to probes designed in the past are summarized.

We recall that the working fluid plays a major role in the probe design. Since we work with GaInSn at room temperature, we used PEEK for the probe tip, which is an electric insulator. The electric insulation of the probe tip component has the big advantage that no effective Seebeck coefficient for the probe tip $S_{e \text {,tip }}$ needs to be determined by calibration, 


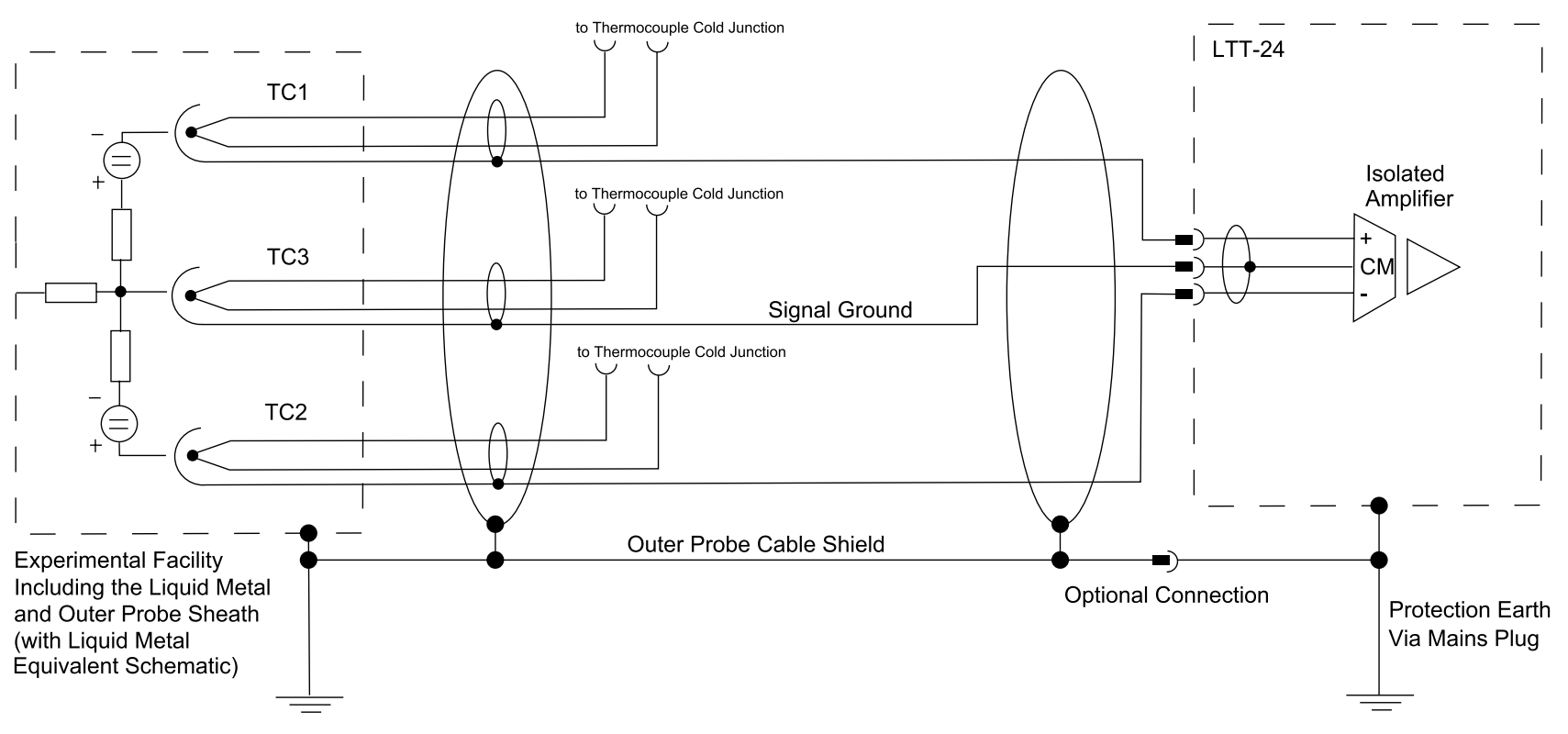

Fig. 6 Sketch of the PMP circuit diagram

i.e., $S_{e, \text { tip }}=S_{\text {GaInSn }}$. For higher temperatures and for liquid sodium, ceramic materials can also be used, see von Weissenfluh and Sigg (1988).

Another advantage of our probe design is the separation of the treatment of the temperature and velocity signal by using ungrounded thermocouples and by measuring the velocity-proportional signal over the thermocouple sheath. All probes in the past used grounded probes, i.e., the velocity-proportional signal was measured over the thermocouplepair. In other words, the velocity-proportional signal rides on top of the temperature signal when using the groundedthermocouple approach. As a consequence, corrections and/ or compensations for both temperature and velocity signals are needed. Alternatively, three-wire thermocouples can be used as well to achieve the same objective as ours of treating both signals separately.

Along the disadvantages of our approach we mention the lower frequency response for the temperature signal when using ungrounded thermocouples. This can be compensated by using thinner thermocouples, but we preferred to stick to thicker thermocouples since extremely thin thermocouples tend to break very easily (during the calibration campaign, the probes were mounted and unmounted several timeshence we opted to use more robust thermocouples). An advantage of past probes compared to ours is that probes used in the past placed the thermocouples on the permanent magnet surface. As a consequence, higher probe sensitivities are theoretically possible. We compensated this effect by using stronger magnets (neodym) than used in the past (AlNiCo).

\section{Calibration of the probe}

\subsection{Wetting issues when using a PMP in a GalnSn flow}

The main drawback when working with GaInSn is the issue regarding its high surface tension, and hence, the wetting of surfaces and objects. In order for a permanent magnet probe to work optimally, we observed that the probe tip needs to be both wetted in a hydrodynamic and an electrical sense. On one hand, if the probe tip is not properly wetted in a hydrodynamic sense, gas pockets may get trapped around the probe tip. The volume-averaged velocity in the surroundings of the probe tip is accordingly lower. This negatively affects the value and the stability probe sensitivity over time. On the other hand, regarding the degree of the electric connection of the three electrodes over the liquid metal, two things could be observed. If no connection at all (i.e., infinite electrical resistance between the electrodes at the thermocouple connectors), the circuit was open and the signal could not be measured. If the electrodes were partially electrically connected (i.e., an electrical resistance of a few $10^{2}$ or $10^{3} \Omega$ ) the external electromagnetic shielding effectiveness was reduced because the shield potential is no longer tightly connected to the liquid metal potential. Furthermore, inductive/capacitive coupling between the electrodes cannot be neglected in that case. For both cases, we observed a constant DC offset of the signal. We believe that the DC offset shift is caused by electromagnetic interferences signals tending to get rectified within the sensitive LTT24 frontends. 
In order to wet a surface with GaInSn, one can either mechanically or chemically wet it (Morley et al. 2008), i.e., to literally rub the liquid metal onto a surface or to clean the surface with an acid prior to expose it to the liquid metal. The chemical method could not be used for this experiment, because in past experiments conducted by partners associated to the LIMCKA, it could be observed that the total removal of acid traces of the liquid metal is hardly possible. This turns out to be a problem when working with rubber seals and plastic objects over longer periods of time (probe tip and test section walls). This is not an issue when working with a pure stainless steel facility and instrumentation.

At the present experiment, the mechanical wetting method was chosen for the test section walls. This method worked fine for the test section judging from differential pressure drop measurements along the test section (not shown in Fig. 1) comparison with empirical correlations and CFD simulations. However, it did not work up to the desired degree for every probe. The wetting had to be enhanced with electro-wetting. This was only necessarily for new probes that had not been used before. Two probes ( $P 1$ and $P 2)$ were mounted to the test section during the commissioning phase of the facility for preliminary tests. As will be shown later, the longer the probes are immersed into GaInSn, the better the wetting of the probe tip. As a consequence, the probe sensitivity was stable at an almost constant value.

The procedure for wetting and quantitatively checking the wetting quality of the probes was the following. First, the probe tip surfaces were thoroughly cleaned with either heptane or iso-propanol. Then, GaInSn was rubbed mechanically with a sponge onto the probe tip up to the point where the contact angle between the surface and the probe tip was observed to be below $90^{\circ}$ and when the probe tip was observed to have a uniform liquid metal thin film on it. The probe to be calibrated was then mounted into the test section and the facility was filled with GaInSn. This did not guarantee an electrical connection between all three electrodes (thermocouple sheaths) for all probes. The electrical connection between the sheaths was assessed by measuring the electric resistance between the electrodes. Interestingly, after once accidentally leaving the multimeter connected to a probe for a few minutes, one could observe that the electric resistance of the probe circuit dropped from a few megaohms to $\sim 35 \Omega$. This phenomenon could be reproduced with all probes. By connecting probe electrodes with a regular $9 \mathrm{~V}$ battery for a few seconds the process could be enhanced and accelerated. The method of wetting an electrically conducting surface with a liquid metal by applying an external voltage between the fluid and the surface is called electrowetting (Eaker and Dicker 2016).

\subsection{Sampling time and sampling rate}

The next questions to be answered are how long and how fast the measurement should take place. Estimates for the integral time scale and the turbulence intensity of the flow at the location of interest are needed (George et al. (1978), Nobach and Tropea (2007)). Unfortunately, it was not possible to measure the turbulence intensity and the autocorrelation function at the inlet of the test section. Any trace of velocity fluctuations at the inlet disappeared in noise. Hence, the sampling time and the sampling rate were estimated from judgement and practical feasibility.

The objective is to obtain a worst-case limiting value for the variability of the estimator for the mean velocity at the channel center of the inlet of the test section. From previous RANS simulations of the contraction preceding the test section (Fig. 1), the turbulence intensity $T u$ was estimated to be $1 \%<T u<2 \%$. For the RANS simulations, a full mesh independency study was performed, all RMS residuals of all equations were below $10^{-5}, y^{+}$values were all below unity, cell expansion factor over the domain was 1.1, a baseline Reynolds stress model was used and all discretization schemes were at least of second order.

For the integral time scale, a very conservative estimate was taken. In the absence of any other options, the integral length scale at the test section inlet was estimated by taking the integral length scale for a fully developed turbulent velocity profile in a channel between two parallel plates. Bailly and Comte-Bellot (2015) indicate that for this geometry the integral length scale $l_{\text {int }}$ can be estimated as 0.6 times the half channel width. For a channel width at the inlet of the test section of $0.04 m, l_{\text {int }}=0.012 \mathrm{~m}$, which is 7.5 times the probe diameter. Of course, the lower the inlet bulk velocity, the slower the integral time scale $t_{\text {int }}$. Hence, the lowest inlet bulk velocity was taken (see Table 2 ) to calculate $t_{\text {int }}$ from $l_{\text {int }}$ by assuming the Taylor hypothesis of frozen turbulence. That is, $t_{\text {int }}=l_{\text {int }} / U_{b}=0.012 / 0.031=0.38 s$.

Considering the constraints imposed by limited timeaccessibility to the lab, considering all the measurement points to be measured, plus the time required to mount and demount the probes, besides the filling and draining time of the facility, a maximum sampling time of $t_{s}=52.43 \mathrm{~s}$ was chosen. This sampling time is predefined by the acquisition system software. The alternatives would have been $t_{s}=26.21 \mathrm{~s}$ or $t_{s}=105 \mathrm{~s}$.

With these values for the turbulence intensity and the integral time scale, a sampling time $t_{s}$ can be estimated for extended confidence interval of $95.45 \%$ (Nobach and Tropea et al. 2007) as

$\epsilon=2 \cdot \sqrt{\frac{2 t_{i n t}}{t_{s}}} \cdot T u=0.32 \%$ 
The value for $\epsilon$ for the maximum inlet bulk velocity using the same procedure is $\epsilon=0.12 \%$. Both values seem acceptable considering all the constraints related to this measurement tasks.

For measuring mean quantities, the needed sampling rates are relatively low. For this case, a sampling rate of $f_{s}=1.30 \mathrm{~Hz}$ would have been enough. Nevertheless, the decimation rate of the LTT24 was still chosen to be $f_{s}=5000 \mathrm{~Hz}$ considering the optimum operation of the LTT24 regarding its electromagnetic interference canceling routines.

\subsection{Calibration procedure}

The calibration task consists on relating the measured voltage $V_{S S}$ with an actual velocity $u$ under isothermal conditions. An in situ calibration is always preferred. Due to the geometry of this particular experiment, an in situ calibration was not feasible. Hence, all probes were sequentially mounted and calibrated at the test section inlet ( $P 1$ in Fig. 1), where by design, the velocity profile could be assumed as constant. Taking the measured value from the flow meter and the inlet channel dimensions, one could obtain a bulk inlet velocity $U_{\text {bulk }}$ from the continuity relation. This assumption seems to be valid within experimental uncertainty, see Fig. 7. The velocity profiles were measured on different days and after the calibration. The red band denotes the experimental uncertainty of the measurements. The values for $\mathrm{Re}_{h}=20000$ and 30000 lie above 1, although the probes were actually calibrated at this place for these velocities. This apparent contradiction is explained due to general repeatability effects of the facility and improved wetting effects of the probes at higher velocities (the boundary layer around the probe tip is thinner, i.e., the volume-averaged velocity is higher).

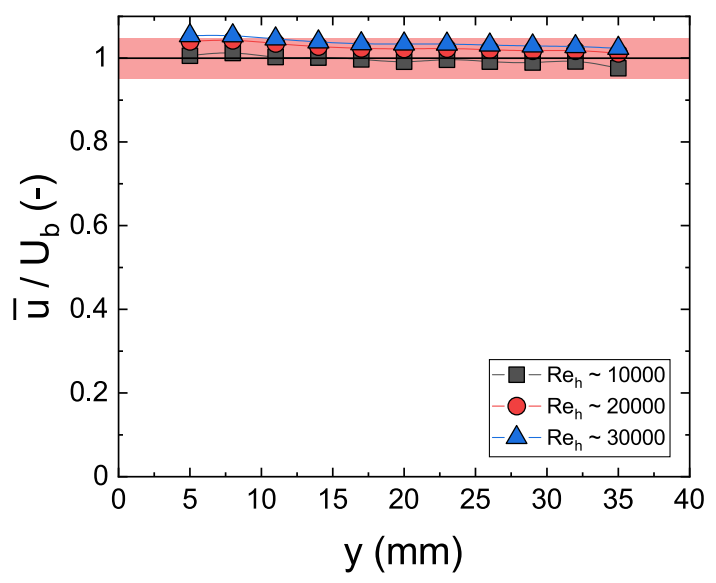

Fig. 7 Test section inlet profiles for three different Reynolds numbers. The red band denotes a 5\% interval within which all measurement points fall into their extended experimental uncertainty
A slight tilt of the velocity profile can be observed toward the low values of $y$ in Fig. 7 as well. Looking at Fig. 1, it seems that the influence of the $90^{\circ}$ bend with vanes was not totally corrected by the three grid stages and the honeycomb in the settling chamber. The pressure drop in the settling chamber had to be limited to the relatively low pressure head of the pump. Hence, screens or grids with the lowest possible solidity were chosen, but guaranteeing a value below the jet coalescence limit (Groth \& Johansson, 1988). After commissioning the facility, the pump pressure head was found to be much higher than indicated in the data sheet. This is most likely to be related to poor wetting of GaInSn along the facility (not the test section), although some issues related to the pump characteristic curve provided by the manufacturer cannot be totally excluded. In the future, screens with a higher pressure drop along the flow conditioning section could be installed. This should guarantee a uniform inlet profile at the test section.

The probes were sequentially calibrated following a systematic procedure in the following order: $P 2, P 3, P 4, P 5, P 6$, $P 1$. Electro-wetting was accidentally found out when calibrating P6. The implemented procedure was: (i) each probe was prepared for calibration (cleaned and mechanically wetted). (ii) The probe to be calibrated was mounted to the test section at position $P 1$ and positioned at the channel center. (iii) The facility was filled with GaInSn ("1 ${ }^{s t}$ run"). The probe signal quality was assessed by monitoring the signal noise to be within its expected noise-level (see Sect. 9.2 in the Appendix) and its instant offset level $( \pm 60 \mathrm{nV})$. This proved to be the case when the electric connection resistance $R$ between the three electrodes was $R<35 \Omega$. If $R>35 \Omega$, the facility was drained and the probe was mechanically wetted again. This proved to be a very time consuming procedure. (iv) The signal $V_{S S}$ was measured for a time period $t_{s}$ (Sect. 4.2) under ambient electromagnetic interference with the pump variable frequency drive once turned off and then on. (v) The pump was set at a rotational frequency $\omega=10 \mathrm{rpm}$ and the flow was let to stabilize. The signal $V_{\mathrm{SS}}$ was measured for a period of time $t_{s}$. (vi) $\omega$ was increased in 10rpm steps until reaching 200rpm. This is called a "run up". Then $\omega$ was decreased back, again every 10rpm, which is called a "run down." For each point, $V_{\mathrm{SS}}$ was measured for a period of time $t_{s}$. (vii) The facility was drained and then filled again. This was repeated two further times for each probe $\left(2^{\text {nd }}\right.$ and $3^{\text {rd }}$ runs), i.e., six calibration curves ("runs") were obtained for every probe. (viii) The probe was demounted from position $P 1$ and mounted into the corresponding position.

Since electro-wetting was found during the calibration of probe $P 6$, an optimized calibration procedure is recommended for future applications and is described in Sect. 4.4.5.

The reason for measuring several "runs" or calibration curves for each probe is that of being able to quantify the repeatability effect regarding the wetting issues of demounting and mounting the probe from the calibration point to the 
actual measuring position. For every "run" the measurement chain was previously warmed up for at least $12 \mathrm{~h}$. The offset was measured before and after all runs. In both measurements for all probes the offset may have varied during the calibration $\pm 0.060 \mu \mathrm{V}$. However, there is no guarantee that during the calibration the offset wandered outside of this threshold.

\subsection{Measured sensitivities}

The measured calibration curves for each probe are shown in Figs. 8, 9, 10, 11 and 12. The calculated sensitivities are shown in Table 1. The uncertainty analysis is presented in detail in the Appendix.

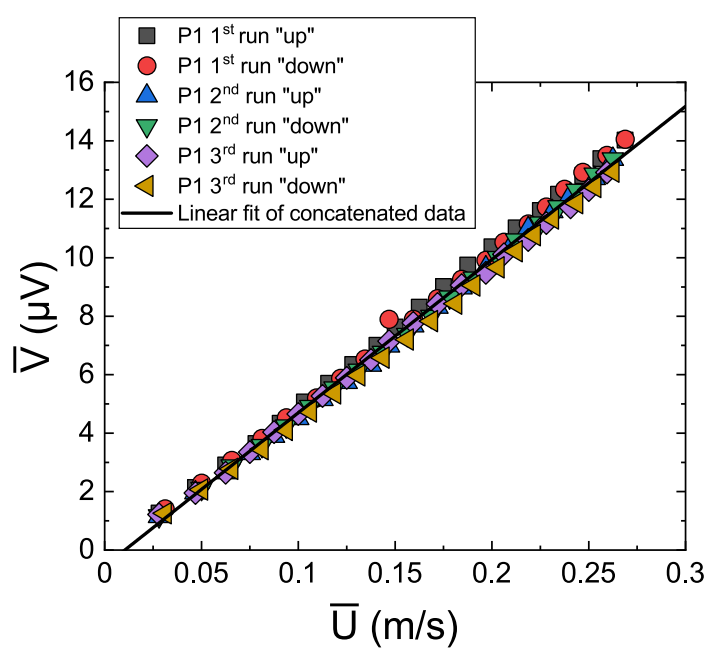

Fig. 8 Calibration curves for probe 1 (P1)

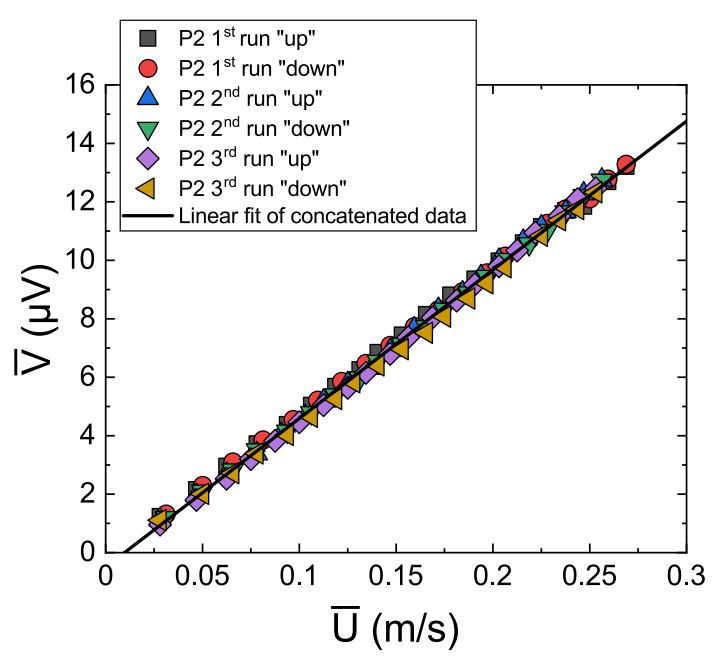

Fig. 9 Calibration curves for probe 2 (P2)

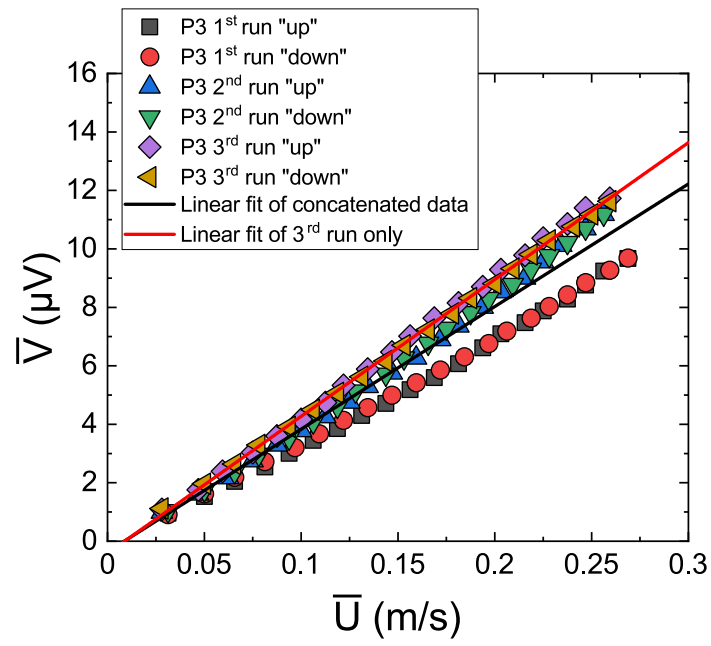

Fig. 10 Calibration curve for probe 3 (P3)

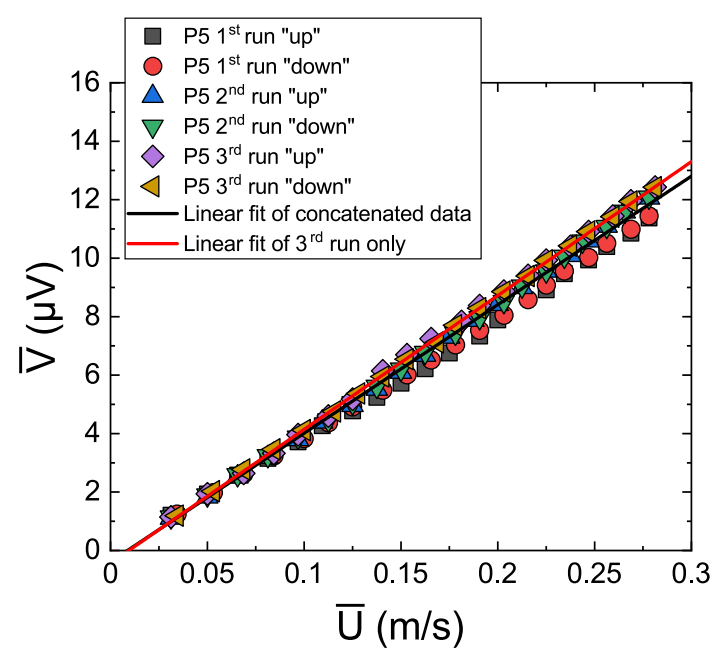

Fig. 11 Calibration curves for probe 5 (P5)

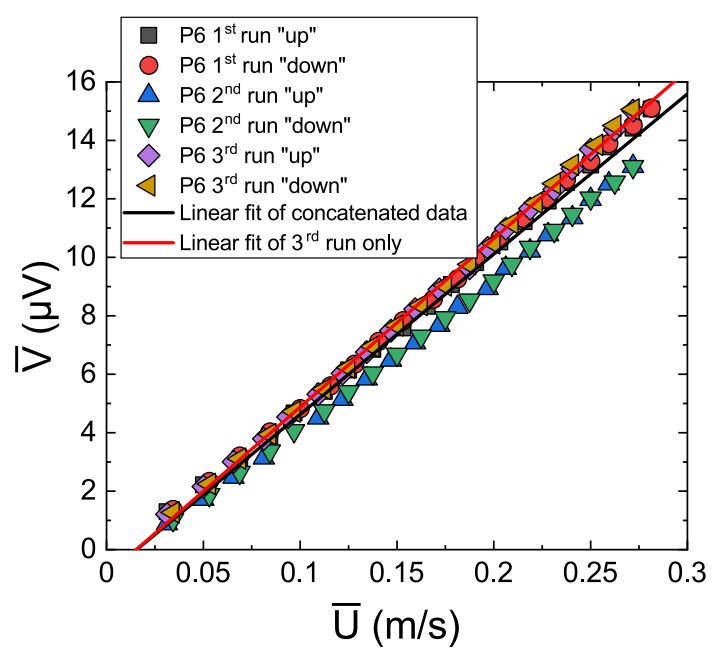

Fig. 12 Calibration curves for probe 6 (P6) 
We recall that for each probe, a total of six calibration curves were measured. For probe $4(P 4)$, however, further runs were necessary due to wetting issues during the calibration campaign. Those probes with similar behavior and measured sensitivities are commented together.

\subsubsection{Probe 1 and probe 2}

Probes 1 and 2 showed very similar results. Probe $2(P 2)$ was the first probe to be calibrated, while probe $1(P 1)$ was the last one. Their measured sensitivities agree well with the theoretical estimate of $K_{\mathrm{th}}=51 \mu \mathrm{V} / \mathrm{m} / \mathrm{s}$ (Sect. 3.4.1). Their behavior during the calibration campaign was also similar in the sense that the variability between runs was stable. No major difference in the sensitivity of the probe can be observed between runs (slope of the curve). This can be explained from the point of view of the quality of the wetting of the probe tip and between the electrodes of the probe. Both probes were mounted to the test section during the commissioning of the facility and the measurement chain, i.e., they were exposed to liquid metal flow for a few weeks before beginning with the calibration campaign. In other words, the liquid metal had enough time to "rub" or "wet" itself onto the probe tip. For these two probes, no electrowetting was needed for the probes to properly work and attenuate external electromagnetic interference influences. Even when the test section was empty, both probes remained wetted. However, $P 1$ was electro-wetted for its first run alone since by that point of the campaign, we had realized this effect. One can observe the minor decrease of its sensitivity along runs. This means that electro-wetting cannot be expected to hold its positive effect between facility draining and filling procedures.

Table 1 Measured sensitivities and respective extended combined uncertainty for each probe

\begin{tabular}{rcc}
\hline & $\bar{K}=K_{\text {conc }}(\mu \mathrm{V} / \mathrm{m} / \mathrm{s})$ & $2 u_{\bar{K}}(\mu \mathrm{V} / \mathrm{m} / \mathrm{s})$ \\
\hline P1-All Runs & 52.41 & 1.72 \\
P2-All Runs & 50.85 & 1.16 \\
P3-All Runs & 41.95 & 6.42 \\
P3-3rd Run only & 46.83 & - \\
P4-All Runs & 23.18 & 8.50 \\
P5-All Runs & 43.97 & 2.91 \\
P5-3rd Run only & 45.78 & - \\
P6-All Runs & 54.82 & 3.99 \\
P6-3rd Run only & 57.62 & - \\
\hline
\end{tabular}

\subsubsection{Probes 3,5 and 6}

These probes showed a similar behavior during the calibration campaign. The measured sensitivities could also be predicted in an order of magnitude sense. For these probes an increasing sensitivity was measured along the calibration campaign. The black linear fit curve corresponds to the concatenated linear fit for all data points, whereas the red line corresponds to concatenated linear fit for the third run (both "up" and "down"). For probes $3(P 3)$ and $5(P 5)$, which were only wetted mechanically, a constant sensitivity increase can be observed along runs. For probe $6(P 6)$, which was the penultimate probe to be calibrated and the first probe to be wetted both mechanically and by electro-wetting, the sensitivity of the second run is lower than that of the other two runs. A possible reason is that for this probe, electro-wetting was done only prior to the first run, i.e., only for this run the sensitivity can be expected to be at its maximum due to optimum wetting. After draining and filling again the facility for the second and third runs, the probe was not wetted again by electro-wetting. This means that the sensitivity increase from the second to the third run may be explained by regular mechanical flow-wetting.

\subsubsection{Probe 4}

This probe shows the lowest measured sensitivity and an atypical behavior. Taking a closer look to Fig. 13a and b one can observe that the probe sensitivity declines after every facility/drain procedure. In fact, during the "third run up" (Fig. 13a) one can observe that the probe suffers a sudden sensitivity decline during the calibration campaign. After noticing this behavior during the experiments, it was decided to demount and re-wet the probe mechanically. The results for this probe after being remounted can be seen in Fig. 13b. The behavior for a decreasing sensitivity can still be observed, however the variability between runs is lower. Since there is no way to know a priori for the actual experimental campaign which sensitivity one should use for this probe, it was decided to take the slope of the concatenated linear fit for the full data set with the penalization of a high standard uncertainty for the sensitivity value. This means that the experimental uncertainty for the results measured with this probe are the highest.

\subsubsection{General comments for all probes}

For all probes, one can observe that the intersection of the linear fits with the ordinate axis is negative. This is physically not possible, since all sensitivity curves were offset within $\pm 60 n V$ corrected before calibration and checked afterward. The most probable explanation for this effect are non-linear effects (Sect. 3.4.2). The velocity-threshold for 

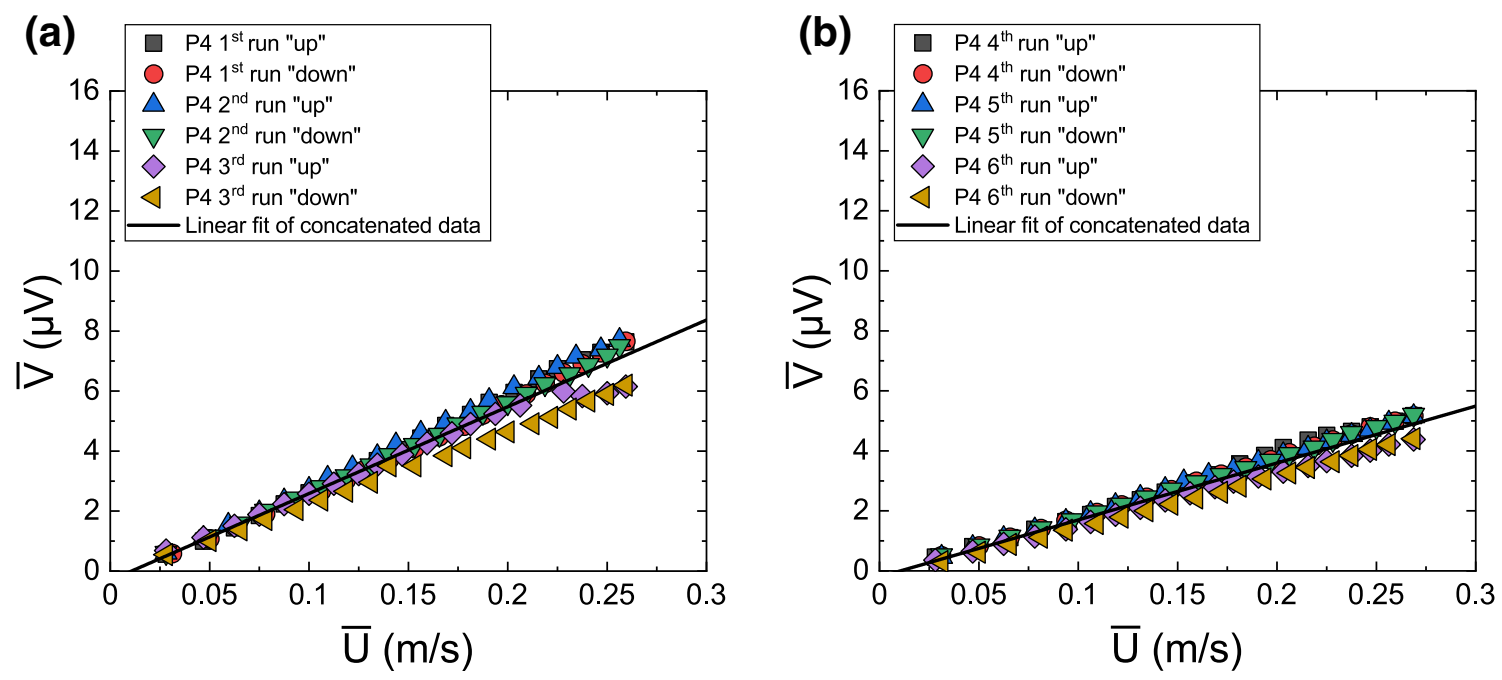

Fig. 13 a Calibration curve for probe 4 (P4). First three runs. b Calibration curves for probe 4-last three runs

the appearance of nonlinear effects is given by the interaction parameter (Eq. 8) and can be estimated as $U_{c}<0.83 \mathrm{~mm} / \mathrm{s}$. As mentioned before, this threshold could not be measure in practice, since the used pump could not be operated for such low flow rates.

The proper thing to do is to have performed a post-calibration of all probes after the actual backward facing step measurement campaign. However, due to time constraints, this was not possible but remains an important task to be done to further assess the quality of the present results.

A further contribution to the good behavior of probes 1 and 2 may have had to do with the cleaning procedure for these two probes. Heptane was used as a cleaning medium for these two probes. However, due to safety concerns manifested by the safety authorities regarding the use and the disposal of heptane on a daily basis, iso-propanol was used instead for the rest of the probes. Whether this may have had an influence or not, is still unclear.

The question whether the sensitivity coefficient for the full data set should be used or only for selected data, e.g., for the third run, must be considered. This has a major influence in the uncertainty estimation. One has to consider that for the actual experimental campaign the facility was not drained during the measurement campaign. Furthermore, every probe was electro-wetted up to a point of an electrode resistance below $35 \Omega$ before the measurement campaign. It would fair to assume that probes 3, 5 and 6 will show a behavior similar to that of probes 1 and 2 after hours of operation. This cannot be said about probe 4 . However, there is no way to really know what the actual sensitivity for each probe for the experimental campaign was. Hence, although it may be an over-conservative approach, the full data set for each probe will be used for both the calculation of the sensitivity and the uncertainty analysis.

\subsubsection{Recommended calibration procedure for the future}

As already mentioned, electro-wetting was accidentally found at rather the end of the calibration, hence its inclusion is not stated in the calibration procedure described in Sect. 4.3. The only difference between the original and the recommended calibration procedure is step (iii), i.e., the step related to the quantitative assessment of the quality of the electric connection between the electrodes and what to do in the case it needs to be improved: in the case that the electrical connection between the three electrodes is measured to be above the defined target $R$, instead of draining the facility and mechanically re-wetting the probe mechanically, we recommend to apply electro-wetting with a regular $9 \mathrm{~V}$ battery until reaching the target $R$. Of course, the target $R$ will depend on the measurement chain. In our case, it happened to be $R<35 \Omega$.

\section{Conclusions}

New permanent magnet probes were successfully developed, adapted and calibrated. Although limitations to this measurement technique exist, we are confident that the designed probes fulfill the requirements for the simultaneous and local measurement of mean and fluctuating velocities and temperatures in a confined vertical backward facing step with a liquid metal as a working fluid. However, due to time and minor technical constraints related to the experimental facility, only mean velocities (time-averaged) are treated in this paper.

The main conclusions of this work are summarized next.

- Permanent magnet probes (PMP) offer advantages compared to ultrasound Doppler velocimetry (UDV) for the 
study of turbulence related quantities, although the technical challenges to be overcome for the practical use of PMP are not minor.

- The used working fluid, the eutectic alloy GaInSn, offers advantages compared to other liquid metals (sodium, lead, lead-bismuth) for instrumentation development regarding safety and handling.

- Due to its very low kinematic viscosity, relatively low velocities are needed to achieve high Reynolds numbers. This means that lower signals are induced in a permanent magnet probe compared to sodium flows for the same Reynolds number.

- As expected, assuming proper wetting of surfaces, design guidelines for the design of wind- or water-tunnels can be transferred to GaInSn facilities. Material compatibility issues need to be taken into account.

- When choosing an electrical insulator for the probe tip material, complex temperature corrections and calibrations are not necessary.

- The theoretical estimations for the probe sensitivity showed good agreement with the measurements.

- The measured probe sensitivity showed - as expected a linear behavior for the given range. Due to technical constraints, the theoretical threshold for the appearance of nonlinear effects in the lower range bound was not validated.

- Due to the high surface tension of GaInSn, wetting issues of the probe tip must be taken into consideration during the whole process of the instrumentation development and use and uncertainty analysis.

- Hydrodynamic wetting of the probe tip alone does not guarantee by itself a good performance of the probe.

- A good electric connection between the liquid metal and the probe electrodes must be guaranteed for the probes to work properly.

- A hydrodynamic wetting condition does not imply immediately a sufficiently good electric connection between the liquid metal and the probe electrodes. At the same time, a good electrical connection between the probe electrodes does not imply directly a good hydrodynamic wetting.

- The experimental facility pumping system should be designed in such a way, that it does not emit too much electromagnetic disturbances. If possible, digital variable frequency drives should be avoided. However, with a careful electromagnetic interference strategy, these influences can be suppressed down to a minimum.

- In situ calibrations for the probes are preferred. This was not possible for the present test section, due to its geo- metrical characteristics. This is a major argument in favor of UDV for this kind of geometry.

- The calibration of the probe in an external calibration facility should be associated with similar uncertainty as for the proposed calibration strategy.

- Assuming a proper wetting procedure of the probe after a few hours of running time of the facility, the probe sensitivity reaches a stable and nearly constant value.

- As expected, the main uncertainty contributor for the measured probe sensitivities is related to the wetting issues.

\section{Appendix}

\section{Fluid properties and facility operational range}

Table 2 Facility operational range for different parameters

\begin{tabular}{|c|c|c|}
\hline & Minimum value & Maximum value \\
\hline$T_{\text {ref }}=T_{\text {inlet }}\left({ }^{\circ} \mathrm{C}\right)$ & 15 & 32 \\
\hline$\dot{V}(1 / \mathrm{s})$ & 0.1 & 2.0 \\
\hline $\mathrm{U}_{\text {bulk, inlet }}(\mathrm{m} / S)$ & 0.031 & 0.625 \\
\hline$U_{\text {bulk, outlet }}(\mathrm{m} / \mathrm{S})$ & 0.016 & 0.313 \\
\hline$\dot{m}(\mathrm{~kg} / \mathrm{S})$ & 0.64 & 12.82 \\
\hline$\dot{q}\left(\mathrm{~W} / \mathrm{m}^{2}\right)$ & - & 14985 \\
\hline $\operatorname{Re}_{h}$ & 3813 & 76256 \\
\hline $\operatorname{Pr}$ & 0.036 & 0.032 \\
\hline $\mathrm{Pe}_{h}$ & 128 & 2557 \\
\hline $\mathrm{Ri}_{h}$ & 0.0028 & 1.14 \\
\hline
\end{tabular}

$T_{\text {ref }}$ is the test section inlet temperature (dependent on the pump rotational speed), $\dot{V}$ is the volume flow rate, $\dot{m}$ the mass flow rate, $\dot{q}$ is the heating plate heat flux input, $R e_{h}$ is the Reynolds number based on the step height and the inlet bulk velocity taking the fluid properties at $25^{\circ} \mathrm{C}$, Pr is the Prandl number evaluated in the $T_{r e f}$ range, $P e_{h}$ is the Peclet number taking $\operatorname{Pr}$ at $25^{\circ} C$ and varying $R e_{h}$ over its range and $R i_{h}$ the Richardson number varying $R e_{h}$ over its range and taking $\dot{q}$ at its maximum value and the fluid properties at $25^{\circ} \mathrm{C}$. The Richardson number was calculated as usually defined for these cases:

$R i_{h}=\frac{g \beta \Delta T h}{U_{b}^{2}}$, with $\Delta T=\frac{\dot{q} h}{\lambda}$

where $g$ is the gravitational acceleration, $\beta$ the volumetric expansion, $\Delta T$ the characteristic temperature difference between the heating plate and bulk flow due to the input heat flux $\dot{q}, \lambda$ the thermal conductivity at the reference temperature at the inlet, $h$ the step's height and $U_{b}$ the velocity of the bulk flow at the inlet. 
Table 3 Thermophysical properties of the working fluid galliumindium-tin (GaInSn) taken from Plevachuk et al. (2014)

\begin{tabular}{llll}
\hline & $\begin{array}{l}\text { Gallium-Indium- } \\
\text { Tin GaInSn }\end{array}$ & Sodium Na & Water $\mathrm{H}_{2} \mathrm{O}$ \\
\hline$T_{\text {ref }}\left({ }^{\circ} \mathrm{C}\right)$ & 25 & 200 & 25 \\
$\rho\left(\mathrm{kg} / \mathrm{m}^{3}\right)$ & 6412 & 903 & 997 \\
$\mu(\mathrm{mPa} \mathrm{s})$ & 2.10 & 0.45 & 0.89 \\
$\nu\left(\mathrm{m}^{2} / \mathrm{s}\right)$ & $0.32 \times 10^{-6}$ & $0.50 \times 10^{-6}$ & $0.89 \times 10^{-6}$ \\
$c_{p}(\mathrm{~J} / \mathrm{kg} \mathrm{K})$ & 400 & 1343 & 4218 \\
$k(\mathrm{~W} / \mathrm{kg} \mathrm{m})$ & 25.06 & 81.94 & 0.68 \\
$\alpha\left(\mathrm{m}^{2} / \mathrm{s}\right)$ & $9.78 \times 10^{-6}$ & $67.57 \times 10^{-6}$ & $0.16 \times 10^{-6}$ \\
$\operatorname{Pr}(-)$ & 0.034 & 0.007 & 5.523 \\
$\gamma(\mathrm{N} / \mathrm{m})$ & 0.59 & 0.19 & 0.07 \\
$\sigma(1 / \Omega \times m)$ & $3.25 \times 10^{6}$ & $7.70 \times 10^{6}$ & $5.49 \times 10^{-6}$ \\
\hline
\end{tabular}

For comparison, the properties for liquid sodium (Sobolev (2011) and water (NIST) are also shown

$\rho$ is the density, $\mu$ is the dynamic viscosity, $v$ is the kinematic viscosity, $c_{p}$ is the specific heat at constant pressure, $k$ is the thermal conductivity, $\alpha$ is the thermal diffusivity, $\gamma$ is the surface tension and $\sigma$ is the electric conductivity.

\section{Theoretical estimation of the noise level due to thermal and flicker noise}

When designing a low-noise measurement system, it is important to roughly estimate a priori what the theoretical minimum possible noise level may be due to intrinsic physical phenomena, i.e., thermal and flicker noise.

Regarding the thermal noise level limits of the measurement system, the theoretical Johnson noise level $n_{\text {Johnson }}$ can be calculated as

$n_{\mathrm{Johnson}}=\sqrt{4 k_{B} T R \Delta f}$

where $k_{B}$ is the Boltzmann constant, $T$ is the reference absolute temperature, $R$ is the electric resistance of the circuit and $\Delta f$ is the bandwidth to be analyzed.

Substituting $k_{B}=1.380649 \times 10^{-23} \mathrm{~J} / \mathrm{K}, T=298.15 \mathrm{~K}$, $R=35 \Omega$ and $\Delta f=500 \mathrm{~Hz}$ one can calculate the noise level due to electronic thermal effects alone along the thermocouple sheaths to be $n_{\text {Johnson }}=16.97 n V$. For $\Delta f=200 \mathrm{~Hz}$ this value reduces to $n_{\text {Johnson }}=10.74 n V$. The used value for $R$ is a representative value for the measured electric resistance of the probe circuit, i.e., liquid metal-to-LTT24 system (see Sect. 4.1).

However, in the low frequency range, the so-called flicker noise or $1 / f$ noise is the major noise contributor. Although there is no analytical expression to account for the flicker noise, it is possible to roughly estimate a worst-case scenario for its contribution together with the thermal noise contribution for a given bandwidth. It does not however consider the decline of the flicker noise at higher frequencies. If one experimentally determines the noise $n_{0}$ of the measurement system within the measured bandwidth $\Delta f_{f l}$ for the flicker noise contribution, one can estimate a theoretical worst-case scenario noise level $n_{t h}$ for an arbitrary frequency range with cutoff frequency $f_{\text {cutoff }}$. The expression reads,

$n_{\mathrm{th}}=\sqrt{\frac{f_{\text {cutoff }}}{\Delta f}} n_{0}$

Replacing the measured values for the present measurement chain under harsh conditions of $n_{0}=0.035 \mu \mathrm{V}=35 \mathrm{nV}$ for $\Delta f_{f l}=9.98 \mathrm{~Hz}$ one obtains for a cutoff frequency of $f_{\text {cutoff }}=500 \mathrm{~Hz}$ that $n_{\text {th }}=0.247 \mu V=247 n V$. The value for the bandwidth $\Delta f_{f l}=9.98 \mathrm{~Hz}$ is given by the sampling time for these tests $t_{s}=52.43 \mathrm{~s}$. The flicker noise contribution for this sampling time is given by the bandwidth $1 / t_{s} \sim 0.02 \mathrm{~Hz}<f<10 \mathrm{~Hz}$. A representative measured value $n_{\text {exp }}$ for all channels of the LTT24 as a stand-alone system, for $f_{\text {cutoff }}=500 \mathrm{~Hz}$ and under realistic experimental conditions was $n_{\exp } \sim 220 n V$. Now, when connecting the probe to the LTT24 system, a measured representative noise level for the given bandwidth is $n_{\text {exp }} \sim 300 n V$. That is, one can say that we were able to suppress all external interference contributions down to $\sim 80 \mathrm{nV}$. This contribution was mainly due to power-line electromagnetic interference, but not over the measurement system itself, but over the liquid metal. One has to consider that the liquid metal is star-grounded together with all other electronic devices of the facility.

Lower values for $n_{0}$ are indeed technically possible at the expense of thermal drift. However, since the measurement campaigns for one parameter set are relatively long (about 10 h), it was preferred to choose an operational amplifier with better thermal drift capabilities at the expense of a lower noise level.

\section{Uncertainty analysis for the measured sensitivities}

The full uncertainty analysis for the probe sensitivity is presented next making use of the recommended procedure according to the GUM.

The uncertainty contributors for $K$ are (i) the flow meter relative accuracy $s_{\text {rel }, \dot{V}}$, (ii) the accuracy of the area measurement of the inlet section measures $s_{A}$, (iii) the relative statistical uncertainty $s_{\text {rel }, V}$ due to the limited integration time for the determination of $V_{\mathrm{SS}}$ and (iv) the uncertainty $s_{r}$ associated with the repeatability of the experiments. It is assumed that the repeatability uncertainty contributor contains the uncertainty contribution of the measurement system thermal drift and the offset drift. Furthermore all wetting issues due to the existing uncertainty of transferring the measured $K$ at position $P 1$ to the other positions. 
For the sake of simplicity, a type B evaluation for the standard uncertainties of each contributors is made. In a first instance, the definition for the measurement equation for $K$ for an error propagation analysis may seem trivial. But it is actually a tricky question. A worst case scenario is chosen based on Eq. 5 and assuming an isothermal flow. The estimated measurement equation reads,

$\bar{K}=\frac{\Delta V_{\mathrm{SS}}}{\Delta \dot{V}} A$

where $\bar{K}$ is the probe sensitivity calculating using a least square linear fit over the whole data cloud for each probe, $\Delta V_{S S}$ is the maximum estimated range for $V_{S S}, \Delta \dot{V}$ the maximum estimated range for the flow rate $\dot{V}$ and $A$ is the inlet cross section area. These values for each probe are tabulated in Table 4.

This worst case scenario handles the accuracy of the flow meter as a constant value over the whole range of the respective quantities. The flow meter accuracy is then multiplied by the range to obtain the respective standard uncertainty. This is a rough estimation for the uncertainty of these contributors, of course. However, as will be shown, these contributors are almost negligible compared to the repeatability standard uncertainty, mainly due to the wetting issues of the probe (Table 5).

The combined standard uncertainty $u_{\bar{K}}$ for $\bar{K}$ reads
Table 6 Type B standard uncertainty estimation for the repeatability of the calibration

\begin{tabular}{lccc}
\hline & $K_{\min }(\mu \mathrm{V} / \mathrm{m} / \mathrm{s})$ & $K_{\max }(\mu \mathrm{V} / \mathrm{m} / \mathrm{s})$ & $s_{r}(\mu \mathrm{V} / \mathrm{m} / \mathrm{s})$ \\
\hline P1 - All Runs & 50.82 & 53.79 & 0.85 \\
P2 - All Runs & 49.87 & 51.86 & 0.57 \\
P3 - All Runs & 36.50 & 47.63 & 3.21 \\
P3 - 3 ${ }^{\text {rd }}$ Run only & 46.09 & 47.63 & 0.44 \\
P4 - All Runs & 17.00 & 31.71 & 4.25 \\
P5 - All Runs & 40.83 & 45.84 & 1.45 \\
P5 - 3 ${ }^{\text {rd }}$ Run only & 45.84 & 45.72 & 0.04 \\
P6 - All Runs & 51.09 & 58.00 & 1.99 \\
P6 - 3 $3^{\text {rd } \text { Run only }}$ & 57.24 & 58.00 & 0.22 \\
\hline
\end{tabular}

$u_{\bar{K}}=\sqrt{\left(\frac{\partial \bar{K}}{\partial \Delta \dot{V}} s_{\Delta \dot{V}}^{2}\right)^{2}+\left(\frac{\partial \bar{K}}{\partial A} s_{A}^{2}\right)^{2}+\left(\frac{\partial \bar{K}}{\partial \Delta V_{\mathrm{SS}}} s_{\Delta V_{\mathrm{SS}}}^{2}\right)^{2}+s_{r}^{2}}$

where $s_{i}$ are the respective standard uncertainties for each contributor.

For the estimation of the repeatability standard uncertainty a type B evaluation was made as well. The measured sensitivities are shown in Table 6, where $K_{\min }$ is the smallest measured sensitivity for that probe, $K_{\max }$ the largest and $\sigma$ is the standard uncertainty for $K$, with $2 s_{r}=\left(K_{\max }-K_{\min }\right) / \sqrt{3}$

Table 4 Reference values to be substituted into the error propagation analysis for the different uncertainty contributors

\begin{tabular}{|c|c|c|c|c|c|c|c|}
\hline & Unit & Probe 1 & Probe 2 & Probe 3 & Probe 4 & Probe 5 & Probe 6 \\
\hline $\bar{K}$ & $\mu V / m / s$ & 52.41 & 50.85 & 41.95 & 23.18 & 43.97 & 54.82 \\
\hline$\Delta \dot{V}$ & $\mathrm{~m}^{3} / \mathrm{s}$ & \multicolumn{7}{|c|}{0.0009} \\
\hline$A$ & $\mathrm{~m}^{2}$ & \multicolumn{7}{|c|}{$08 \cdot 0.04=0.0032$} \\
\hline$\Delta V_{S S}$ & $\mu V$ & 14.74 & 14.30 & 11.80 & 6.52 & 12.37 & 15.42 \\
\hline
\end{tabular}

Table 5 Equivalent standard deviations for the variables for each probe

\begin{tabular}{|c|c|c|c|c|c|c|c|c|}
\hline & & Unit & Probe 1 & Probe 2 & Probe 3 & Probe 4 & Probe 5 & Probe 6 \\
\hline \multirow{4}{*}{$s_{\Delta \dot{V}}$} & "Accuracy" & $\%$ & \multicolumn{6}{|c|}{0.42} \\
\hline & & $m^{3} / s$ & \multicolumn{6}{|c|}{$3.78 \cdot 10^{-6}$} \\
\hline & $\begin{array}{l}\text { Distribution } \\
\text { factor }\end{array}$ & - & \multicolumn{6}{|c|}{$1 / \sqrt{3}$} \\
\hline & $s_{\Delta \dot{K}}$ & $\mathrm{~m}^{3} / \mathrm{s}$ & \multicolumn{6}{|c|}{$1.09 \cdot 10^{-6}$} \\
\hline \multirow{4}{*}{$s_{\mathrm{A}}$} & Tolerance & $m$ & \multicolumn{6}{|c|}{$0.1 \cdot 10^{-3}$ (for one dimension) } \\
\hline & $\begin{array}{l}\text { Distribution } \\
\text { factor }\end{array}$ & - & \multicolumn{6}{|c|}{ 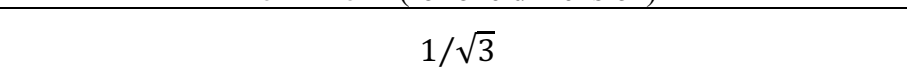 } \\
\hline & $s_{1 \mathrm{D}}$ & $m$ & \multicolumn{6}{|c|}{$57.74 \cdot 10^{-6}$} \\
\hline & $s_{\mathrm{A}}$ & $m^{2}$ & \multicolumn{6}{|c|}{$5.16 \cdot 10^{-6}$} \\
\hline \multirow{4}{*}{$s_{\Delta \mathrm{V}_{\mathrm{SS}}}$} & Precision & $\%$ & \multicolumn{6}{|c|}{0.34} \\
\hline & & $n V$ & 47.17 & 45.77 & 37.75 & 20.86 & 39.57 & 49.33 \\
\hline & $\begin{array}{c}\text { Distribution } \\
\text { factor }\end{array}$ & - & \multicolumn{6}{|c|}{$1 / \sqrt{3}$} \\
\hline & $s_{\Delta V_{S S}}$ & $n V$ & 13.62 & 13.21 & 10.90 & 6.21 & 11.42 & 14.24 \\
\hline
\end{tabular}

All distributions are assumed rectangular. All calculation steps were made with all digits— the results are shown with only two significant digits 
Table 7 Standard uncertainties, combined uncertainty and extended uncertainty $(95.4 \%$ confidence interval)

\begin{tabular}{llcccccc}
\hline & Unit & Probe 1 & Probe 2 & Probe 3 & Probe 4 & Probe 5 & Probe 6 \\
\hline$u_{\Delta \dot{V}}$ & $\mu \mathrm{V} / \mathrm{m} / \mathrm{s}$ & 0.064 & 0.062 & 0.051 & 0.028 & 0.053 & 0.066 \\
$u_{A}$ & $\mu \mathrm{V} / \mathrm{m} / \mathrm{s}$ & 0.085 & 0.082 & 0.068 & 0.037 & 0.071 & 0.088 \\
$u_{\Delta \mathrm{V}_{\mathrm{SS}}}$ & $\mu \mathrm{V} / \mathrm{m} / \mathrm{s}$ & 0.051 & 0.050 & 0.041 & 0.023 & 0.043 & 0.054 \\
$s_{r}=u_{\mathrm{r}}$ & $\mu \mathrm{V} / \mathrm{m} / \mathrm{s}$ & 0.85 & 0.57 & 3.21 & 4.25 & 1.45 & 1.99 \\
$u_{\bar{K}}$ & $\mu \mathrm{V} / \mathrm{m} / \mathrm{s}$ & 0.86 & 0.58 & 3.21 & 4.25 & 1.45 & 1.99 \\
$2 u_{\bar{K}}$ & $\mu \mathrm{V} / \mathrm{m} / \mathrm{s}$ & 1.72 & 1.16 & 6.42 & 8.50 & 2.91 & 3.99 \\
\hline
\end{tabular}

under the assumption of a rectangular distribution of $K$ between $K_{\max }$ and $K_{\min }$.

The individual standard uncertainties, the combined and the extended standard uncertainty are presented in Table 7.

As can be observed in Table 7 and as expected, the uncertainty due to repeatability between runs is the main uncertainty contributor. The repeatability issues are most likely to be related with wetting issues of the liquid metal. It needs to be noted that the repeatability uncertainty contributor was calculated using a conservative approach.

Acknowledgements Dr.-Ing. W. Hering and Prof. Dr.-Ing. R. Stieglitz from INR-KIT for general project support. (ii) O. Albrecht and R. Schmidt from the INR-KIT workshop for mounting and installing the facility and its equipment. (iii) H.J. Brinkmann, Prof. Dr.-Ing. L. Bühler and Dr. C. Courtessole from the MEKKA laboratory at ITES-KIT for their hints during the design and/or the commissioning phase of the facility and the instrumentation. (iv) M. Daubner and F. Fellmoser from the KALLA lab at ITES-KIT for their hints during the commissioning phase of the facility. (v) K. Krauth, D. Kuntz and P.K. Wagner-Nagy from the central workshop at KIT for their support on the design and the manufacturing of the facility and the instrumentation. (v) Dr.-Ing. T. Wondrak, T. Gundrum and Dr. A. Cramer at HZDR, Dr.-Ing. S. Lenk at SAAS GmbH, R. Vollmer from ITES-KIT and Dr.-Ing. F. Arbeiter and G. Schlindwein at INR-KIT for their hints during the troubleshooting of the commissioning phase of the facility and/or the instrumentation. We would also like to thank the referees for their helpful comments and suggestions.

Author's contributions TS contributed to the conception of the experiment and the design and commissioning of the facility, the test section and the instrumentation. SW, JK and MT contributed to the instrumentation and/or measurement chain design and/or manufacturing and/or commissioning. TS planned and prepared the experimental campaign. TS collected, analyzed and interpreted the data. TS wrote the first draft of the manuscript and all authors commented on previous versions of the manuscript. All authors read and approved the final manuscript.

Funding Open Access funding enabled and organized by Projekt DEAL. Financial support was provided by the EU-Horizon 2020 SESAME (Grant agreement ID: 654935) and ESFR-Smart (Grant agreement ID: 754501) projects.

Availability of data and material The datasets generated during and/or analyzed during the current study are available from the corresponding author on reasonable request.

Code availability Not applicable.
Conflicts of interest The authors have no conflicts of interest to declare that are relevant to the content of this article.

Open Access This article is licensed under a Creative Commons Attribution 4.0 International License, which permits use, sharing, adaptation, distribution and reproduction in any medium or format, as long as you give appropriate credit to the original author(s) and the source, provide a link to the Creative Commons licence, and indicate if changes were made. The images or other third party material in this article are included in the article's Creative Commons licence, unless indicated otherwise in a credit line to the material. If material is not included in the article's Creative Commons licence and your intended use is not permitted by statutory regulation or exceeds the permitted use, you will need to obtain permission directly from the copyright holder. To view a copy of this licence, visit http://creativecommons.org/licenses/by/4.0/.

\section{References}

Bailly C, Comte-Bellot G (2015) Turbulence. Springer International Publishing, Cham. https://doi.org/10.1007/978-3-319-16160-0

Barleon L, Mack KJ, Stieglitz R (1996) The MEKKA-facility a flexible tool to investigate MHD-flow phenomena. Technical report FZKA 5821, Forschungszentrum Karlsruhe. https://doi.org/10. 5445/IR/270039825

Cadwallader LC (2003) Gallium safety in the laboratory. Conference: Energy Facility Contractors Group (EFCOG) Safety Analysis Working Group (SAWG) 2003 Annual Meeting, Salt Lake City, UT (US), 06/21/2003--06/27/2003

Cramer A, Eckert S, Gerbeth G (2013) Flow measurements in liquid metals by means of the ultrasonic Doppler method and local potential probes. Euro Phys J Special Topics 220:25-41. https:// doi.org/10.1140/epjst/e2013-01794-2

Cramer A, Varshney K, Gundrum Th, Gerbeth G (2006) Experimental study on the sensitivity and accuracy of electric potential local flow measurements. Flow Meas Instrum 17(1):1-11. https://doi. org/10.1016/j.flowmeasinst.2005.08.006

Davidson PA (2017). An Introduction to Magnetohydrodynamics, 2nd edn. Cambridge University Press, Cambridge. https://doi.org/10. 1017/9781316672853

Eaker CB, Dicker MD (2016) Liquid metal actuation by electrical control of interfacial tension. Appl Phys Rev 3:031103. https://doi. org/10.1063/1.4959898

Eckert S, Cramer A, Gerbeth G (2007) Velocity measurement techniques for liquid metal flows. In: Molokov S, Moreau R, Moffatt HK (eds) Magnetohydrodynamics historical evolution and trends. Springer, Netherlands. https://doi.org/10.1007/978-1-4020-4833-3

Farge M (1992) Wavelet transforms and their applications to turbulence. Annu Rev Fluid Mech 24(1):639-669. https://doi.org/10. 1146/annurev.fl.24.010192.002143

\section{Declaration}


Farge M, Kevlahan N, Perrier V, Goirand E (1996) Wavelets and turbulence. Proc IEEE 84(4):639-669. https://doi.org/10.1109/5. 488705

Fenton AW (1980) How do thermocouples work. Nucl Energy 19(1):61-63

Franke S, Lieske H, Fischer A, Büttner L, Czarske J, Räbiger D, Eckert S (2013) Two-dimensional ultrasound Doppler velocimeter for flow mapping of unsteady liquid metal flows. Ultrasonis 53:691700. https://doi.org/10.1016/j.ultras.2012.10.009

Geddis P, Wu L, McDonald A, Chen S, Clements B (2020) Effect of static liquid Galinstan on common metals and non-metals at temperatures up to $200^{\circ} \mathrm{C}$. Can J Chem 98(12):787-798. https:// doi.org/10.1139/cjc-2020-0227

George WK (2013). Lectures in turbulence for the $21^{\text {st }}$ Century. Available online at http://www.turbulence-online.com/ - last accessed on 11.08.2021.

George WK, Beuther PD, Lumley JL (1978). Processing of random signals. In: Hansen BW (eds) Proceedings of the dynamic flow conference 1978 on dynamic measurements in unsteady flows. Springer, Dordrecht. https://doi.org/10.1007/978-94-009-95659_43

Groth J, Johansson A (1988) Turbulence Reduction by screens. J Fluid Mech 197:139-155. https://doi.org/10.1017/S0022112088003209

Kapulla R (2000) Experimentelle Untersuchung von thermisch stratifizierten und unstratifizierten Mischungsschichten in Natrium und Wasser. Dissertation, ETH Zürich.

Kapulla R, Sigg B, Horanyi S, Hudina M (2000) Local velocity measurements in a thermally-stratified sodium mixing layer using a permanent-magnet probe. Exp Thermal Fluid Sci 20:115-136. https://doi.org/10.1016/S0894-1777(99)00036-9

Knebel JU, Krebs L (1993) Kalibrierung einer Miniatur-Permanentmagnet-Potentialsonde zur Geschwindigkeits- und Temperaturmessung in Natrium. Technical Report Kernforschungszentrum Karlsruhe KfK 5215. https://doi.org/10.5445/IR/270034481

Knebel JU, Krebs L (1994) Calibration of a miniature permanent magnet flowmeter probe and its application to velocity measurements in liquid sodium. Exp Thermal Fluid Sci 8(2):135-148. https:// doi.org/10.1016/0894-1777(94)90041-8

Knebel JU, Krebs L, Müller U (1994) Turbulent convection in buoyant sodium jets. Exp Thermal Fluid Sci 8:149-157. https://doi.org/10. 1016/0894-1777(94)90042-6

Morley NB, Burris J, Cadwallader LC, Nornberg MD (2008) GaInSn usage in the research laboratory. Rev Sci Instrum 79:056107. https://doi.org/10.1063/1.2930813
Nobach H, Tropea C (2007) Fundamentals of data processing. In Tropea C, Yarin A, Foss J (eds) Springer handbook of experimental fluid dynamics. Springer-Verlag, Heidelberg, pp 1399-1417 https://doi.org/10.1007/978-3-540-30299-5

Plevachuk Y, Sklyarchuk V, Eckert S, Gerbeth G, Novakovic R (2014) Thermophysical properties of the liquid Ga-In-Sn eutectic alloy. J Chem Eng Data 59(3):757-763. https://doi.org/10.1021/je400 $882 q$

Ricou R, Vives C (1982) Local velocity and mass transfer measurements in molten metals using an incorporated magnet probe. Int J Heat Mass Transf 25(10):1579-1588. https://doi.org/10.1016/ 0017-9310(82)90036-9

Schulenberg T, Stieglitz R (2010) Flow measurement techniques in heavy liquid metals. Nucl Eng Des 240(9):2077-2087. https:// doi.org/10.1016/j.nucengdes.2009.11.017

Sobolev V (2011) Database of thermophysical properties of liquid metal coolants for GEN-IV. BLG Open Report Series of the Belgian Nuclear Research Centre, Dec 2011. Report number BLG-1069.

Tihon J (2010) Penkavova V, Pantzali M. The effect of inlet pulsations on the backward-facing step flow. European J Mech B/Fluids 29:224-235. https://doi.org/10.1016/j.euromechflu.2010.02.001

Vogt T, Horn S, Aurnou JM (2021) Oscillatory thermal-inertial flows in liquid metal rotating convection. J Fluid Mech 911:A5. https:// doi.org/10.1017/jfm.2020.976

von Weissenfluh T, Sigg B (1988) Experience with permanent magnet probes for the measurement of local velocities in liquid metals. In: Unger Y, Mond M, Branover H (eds) Liquid metal flows: Magnetohydrodynamics and Application. https://doi.org/10.2514/4. 865862

von Weissenfluh T (1985) Probes for local velocity and temperature measurements in liquid metal flow. Int J Heat Mass Transf 28(8):1563-1574. https://doi.org/10.1016/0017-9310(85)90258-3

Wetzel T (2015) Instrumentation. In: OECD/NEA handbook on leadbismuth eutectic alloy and lead properties, materials compatibility, thermal-hydraulics and technologies, Nuclear Energy Agency, pp 731-839.

Zürner T, Schindler F, Vogt T, Eckert S, Schumacher J (2020) Flow regimes of Rayleigh-Bénard convection in a vertical magnetic field. J Fluid Mech 894:A21. https://doi.org/10.1017/jfm.2020.264

Zürner T, Vogt T, Resagk C, Eckert S, Schumacher J (2018) Local Lorentz force and ultrasound Doppler velocimetry in a vertical convection liquid metal flow. Exp Fluids 59:3. https://doi.org/10. 1007/s00348-017-2457-0

\section{Authors and Affiliations}

\section{Thomas Schaub ${ }^{1} \odot$. Sascha Wüstling ${ }^{2} \cdot$ Joachim Konrad $^{3} \cdot$ Michael Tasler $^{4}$}

1 Institute for Neutron Physics and Reactor

Technology (INR), Karlsruhe Institute of Technology

(KIT), Hermann-von-Helmholtz-Platz 1,

Eggenstein-Leopoldshafen, Germany

2 Institute for Data Processing and Electronics (IPE), Karlsruhe Institute of Technology (KIT), Hermann-von-Helmholtz-Platz 1, Eggenstein-Leopoldshafen, Germany
3 Technikhaus (TEC), Karlsruhe Institute of Technology (KIT), Hermann-von-Helmholtz-Platz 1, Eggenstein-Leopoldshafen, Germany

4 Labortechnik Tasler GmbH, Friedrich-Bergius-Ring 15, 97076 Würzburg, Germany 